Apidologie, 1976, 7 (1), 1-38.

\title{
LES INSECTES POLLINISATEURS DE LA FÉVEROLE D'HIVER (VICIA FABA EQUINA L.) ET LA POLLINISATION DES PLANTES MÂLE-STÉRILE EN PRODUCTION DE SEMENCE HYBRIDE
}

\author{
Die die Winter-Pferdebohne (Vicia faba equina $L$.) \\ bestäubenden Insekten und die Bestäubung der männlich-sterilen \\ Pflanzen bei der Erzeugung von Hybridsamen
}

\section{J.N. TASEI}

Laboratoire d'Éthologie et d'Écologie des Insectes I.N.R.A.

S.A.P.F., 86600 Lusignan

\section{SUMMARY}

POLLINATORS OF WINTER FIELD BEANS (Vica faba equina L.) and Pollination of Male-sterile plants for hyBrid seed produrtion.

The insect pollinators of winter field beans (Vicia faba) have been studied for four years mainly at Lusignan (department of Vienne) Fields included both classical crops and sowings of alternate strips of male-fertile and male-sterile plants.

Honeybees make up $80 \%$ of the Apoidea fauna, Terrestribombus $8,4 \%$, Eucera tuberculata $4,0 \%$, Hortobombus $3,5 \%, B$. lapidarius $2,1 \%$, Anthophora acervorum $0,7 \%$, B. pratorum $0,4 \%$.

Terrestribombus bite through the corolla and in 90-95\% of instances do not pollinate the flowers (" negative") visits), Apis mellifica and $B$. pratorum are $80-95 \%$ " negative ", $B$. agrorum and B. lapidarius $10-15 \%$. Eucera, Anthophora, Hortobombus are $100 \%$ pollinators, they are responsible for the major part of the seed produced on the female line. Pollen collecting honeybees are attracted most by the male-fertile line and visit male-sterile plants only by mistake. The other Apoidea show no striking preference for either one parent line. Placing hives in hybrid seed bean crops is probably worthless because of the bees variable behaviour, their lack of interest in male-sterile plants and their frequent negative foraging (used holes pierced by Terrestribombus). 


\section{RÉSUMÉ}

La faune pollinisatrice de la féverole d'hiver (Vicia faba) est étudiée pendant 4 ans principalement dans la région de Lusignan (département de la Vienne). Les champs observés sont des cultures mixtes composés de 2 lignées de plantes mâle-stérile et mâle-fertile semées en bandes alternées ou bien des cultures classiques.

L'Abeille domestique représente près de $80 \%$ de la faune d'apoïdes, les Terrestribombus : 8,4 \%, Eucera tuberculata: $4 \%$, les Hortobombus : $3,5 \%$, Bombus lapidarius : $2,1 \%$, Anthophora acervorum : $0,7 \%$ et $B$. pratorum : $0,4 \%$.

Les Terrestribombus percent les corolles et ne pollinisent pas les fleurs dans $90-95 \%$ des cas (visites " négatives "). Apis mellifica est " négative " dans $80-95 \%$ des cas ainsi que $B$. pratorum. B. agrorum et B. lapidarius ne font que 10-15\% de visites " négatives 》. Eucera, Anthophora et les Hortobombus sont pollinisateurs à $100 \%$ et c'est à ces dernières espèces que l'on doit la majorité de la production de semence récoltée sur le parent femelle.

Les abeilles domestiques butineuses de pollen sont beaucoup plus attirées par les lignées mâle-fertile et ne visitent les lignées mâle-stérile que par erreur. Les autres apoïdes n'ont pas de préférence notable pour l'une ou l'autre des lignées parentales. L'Abeille domestique est 20 à 30 fois moins efficace que les Hortobombus. Il ne semble pas que dans le cas de culture mixte de féverole l'apport de ruches soit rentable du fait de la variabilité du comportement de l'Abeille domestique, de sa très faible attirance pour les plantes mâle-stérile et de son comportement “ négatif " fréquent (utilisation des trous percés par les Terrestribombus).

\section{INTRODUCTION}

La littérature concernant la pollinisation de Vicia faba L. (fève et féverole) est assez abondante. La revue de Free (1970) sur ce sujet et les travaux du danois Poulsen (1973) font l'inventaire des agents responsables de la fécondation chez $V$. faba et soulignent l'importance des bourdons à langue longue ainsi que la variabilité du comportement de l'abeille domestique souvent détournée de la voie de butinage normal par les trous que percent à la base des fleurs certains bourdons à langue courte. Par ailleurs Bond et HAwKins (1967), sont les seuls auteurs à relater des observations sur le comportement des insectes butinant dans un champ de production de semence hybride composé de lignes de plantes “ mâle » et de lignes de plantes “ femelle » alternées. Les plantes " mâle " appartiennent à une lignée possédant des fleurs hermaphrodites (mâle-fertile) tandis que la lignée “ femelle " produit des fleurs dépourvues de pollen fertile (mâle-stérile). On récolte la semence hybride sur la seule lignée " femelle ». Bond et Hawkins jugent que l'abeille domestique semble très peu efficace pour polliniser les fleurs mâle-stérile. Ils estiment que les fleurs à anthères stériles ont beaucoup moins de chances que les fleurs ordinaires, de produire des gousses. Seule une faible partie de la faune fréquente alternativement les deux types de plantes, transportant ainsi le pollen fertile sur les fleurs mâle-stérile. 
Or l'application de recherches sur la stérilité-mâle de la féverole entreprises en France et en Angleterre par Berthelen, Bond et Picard, doit permettre la création de variétés hybrides plus productives que les populations et variétés actuelles. Delaude et Audy (1970) prévoient donc un regain d'intérêt chez les producteurs français, pour cette plante, qui en dépit de la richesse en protéines de sa graine, et de ses qualités agronomiques, ne représente qu'une faible partie de la production des légumineuses fourragères. En prévision de ce bouleversement il paraissait souhaitable d'entreprendre une étude sur les insectes pollinisateurs de la féverole en France, et sur les possibilités de production de semence hybride dans les conditions de la pratique agricole.

Dans cet article j’expose les connaissances acquises en quatre ans sur le spectre des espèces pollinisatrices de la féverole et leur efficacité respective. J'étudie particulièrement l'influence de la stérilité-mâle sur le comportement de butinage des abeilles sauvages et de l'abeille domestique, et sur le transport du pollen de la lignée mâle-fertile à la lignée mâle-stérile.

\section{MATÉRIEL ET MÉTHODE}

La plupart des observations se déroulent sur les champs de la Station d'Amélioration des Plantes fourragères de Lusignan (Vienne) de 1971 à 1974. Chaque année des lignées de féverole d'hiver différentes sont installées sur des surfaces de 0,8 à 6,5 hectares. Il est à noter que les lignées mâle-stérile utilisées n'ont jamais une pureté totale et que le phénomène de restauration spontanée de fertilité se manifeste chez 7 à 20 pour cent des plantes supposées mâlestérile. Ce taux de restauration varie selon la lignée c'est-à-dire selon l'année (Tableau 1).

TABL. 1. - Champs de production de semence de fêverole d'hiver avec utilisation de plantes mâle-stérile et mâle-fertile à Lusignan.

ТАВ. 1. - Feld für die Samenerzeugung der Winter-Pferdebohne unter Verwendung von männlichsterilen und männlich-fertilen Pflanzen. Lusignan.

\begin{tabular}{|c|c|c|c|c|c|c|c|c|}
\hline \multirow[t]{2}{*}{$\begin{array}{l}\text { Année } \\
\text { Juhr }\end{array}$} & \multicolumn{2}{|c|}{$\begin{array}{l}\text { Nombre de } \\
\text { lignes adjacentes } \\
\text { d'une même lignée } \\
\text { Zahl der nebencinander } \\
\text { liegenden Reihen d. } \\
\text { gleichen Stanmes }\end{array}$} & \multicolumn{2}{|c|}{$\begin{array}{l}\text { Lignées } \\
\text { Stänme }\end{array}$} & \multirow{2}{*}{$\begin{array}{c}\text { Pourcentage } \\
\text { de restauration } \\
\text { de fertilité } \\
\text { parmi } \\
\text { les plantes } \\
\text { mâle-stérile } \\
\text { Prozent satz d. } \\
\text { wiederher- } \\
\text { gestellten } \\
\text { Fruebtharkeit } \\
\text { männl. steriler } \\
\text { Pfianzen }\end{array}$} & \multirow[t]{2}{*}{$\begin{array}{c}\text { Superficie } \\
\text { (ha) } \\
\text { Fäche } \\
\text { (ha) }\end{array}$} & \multirow[t]{2}{*}{$\begin{array}{l}\text { Dates de } \\
\text { début et } \\
\text { de fin de } \\
\text { floraison } \\
\text { Datum von } \\
\text { Anfang und } \\
\text { Ende der } \\
\text { IBlutezeit }\end{array}$} & \multirow[t]{2}{*}{$\begin{array}{c}\text { Apport } \\
\text { de ruches } \\
\text { (Nombre } \\
\text { de ruches) } \\
\text { Zahl der } \\
\text { aufgestellten } \\
\text { Völker }\end{array}$} \\
\hline & $\mathbf{F}^{1}$ & $S^{2}$ & $F^{3}$ & S & & & & \\
\hline 1971 & 4 & 4 & $29 \mathrm{D}$ & $29 \mathrm{D}$ & 9 & 0,8 & $27.4 / 30.5$ & 0 \\
\hline 1972 & 6 & 6 & $29 \mathrm{D}$ & $29 \mathrm{D}$ & 20 & 2 & $8.5 / 14.6$ & 0 \\
\hline 1973 & 6 & 6 & $972 \mathrm{D}$ & $972 \mathrm{D}$ & 11 & 6,5 & $9.5 / 6.6$ & 4 \\
\hline 1974 & 6 & 6 & $48 \mathrm{~B}$ & L, 6 & 7 & 4,5 & $25.4 / 4.6$ & 4 \\
\hline
\end{tabular}

1. Plantes mâle-fertile.

2. Plantes mâle-stérile.
1. Männlich-fertile Pflanzen.

2. Männlich-sterile Pfanzen. 
Les deux lignées en présence sont semées sur le terrain en bandes alternées séparées par des allées de $0.8 \mathrm{~m}$. Chaque bande est composée de 4 ou 6 liqnes distantes les unes des autres de $0,4 \mathrm{~m}$. Le peuplement est en moyenne 20 à 30 plantes par $\mathrm{m}^{2}$ ce qui représente environ 50 à 80 tiges au $\mathrm{m}^{2}$. En 1971 les bandes sont interrompues tous les $24 \mathrm{~m}$ par des espaces vides de $2 \mathrm{~m}$ afin de modifier le comportement des insectes butineurs (Fig. 1). Par ailleurs, de 1972 à 1974 des ruches d'abeilles munies de trappes à pollen sont apportées dans la culture expérimentale (Tableau 1). Toutes les lignées étant des féveroles d'hiver les floraisons sont assez précoces et s'étalent du 25 avril au 14 juin (dates extrêmes).

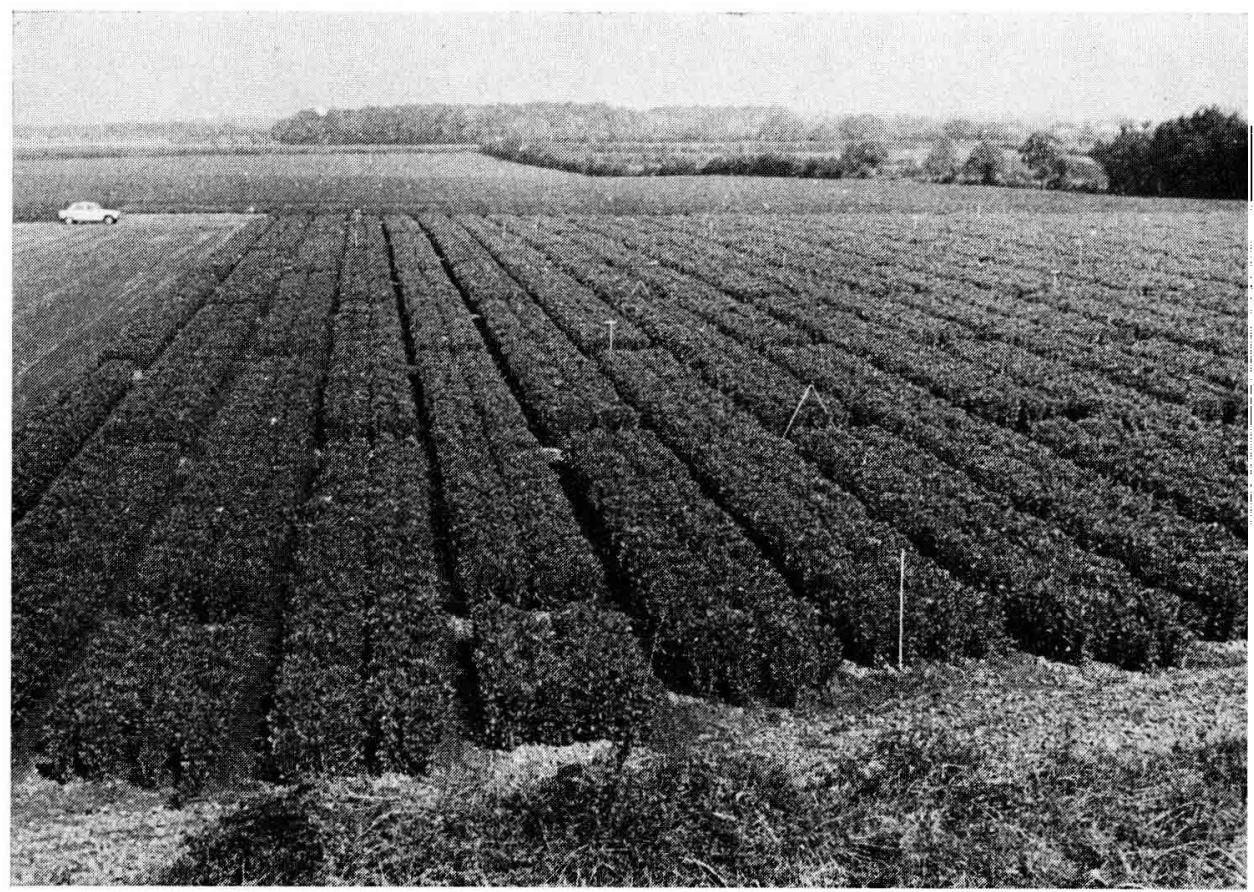

FIG. 1. - Champ de féverole (Vicia faba equina $\mathrm{L}_{\text {.) }}$, avec alternance de bandes de plantes mâle-stërile et mâle-fertile, observé à Lusignan en 1971 (Photo A. Delaude).

Aвв. 1. ... Bohnenfeld (Vicia faba equina L.) mit abwechselnden Reihen von männlich-sterilen. und männlich-fertilen l'flanzen, beobachtet 1971 in Lusignan. (A. Delaude phot.)

En 1973, 19 champs de féverole répartis dans 8 départements servent à établir un premier inventaire de faune ayant une valeur générale. Ces champs de féverole en culture classique ou en production d'hybride ont une superficie moyenne de 3 hectares.

Fn 1974, grâce à la F.N.A.M.S. ' et à certains Établissements multiplicateurs, on installe dans 7 départements une dizaine de champs sur lequels on étudie la mise à graine en fonction de la largeur des bandes mâle stérile. Chacun des champs est composé de 3 parties représentant chacune un dispositif de répartition des lignées différent :

- Premier dispositif : alternance de bandes mâle-fertile de $2,5 \mathrm{~m}$ de large et de bandes mâle-stérile de $2,5 \mathrm{~m}$ de large.

- Deuxième dispositif : les bandes mâle-stérile ont $5 \mathrm{~m}$ de large, les bandes mâle-fertile conservent la largeur $2,5 \mathrm{~m}$. 
- Troisième dispositif : les bandes mâle-stérile ont $7,5 \mathrm{~m}$ de large, les bandes mâlefertile conservent la largeur $2,5 \mathrm{~m}$.

Sept des 10 champs sont des croisements identiques : lignées 48 B et S 45.

$$
1^{0} \text { - Étude de la floraison }
$$

On évalue la densité de fleurs en faisant 2 fois par semaine, toujours à la même heure, un dénombrement des corolles épanouies sur 4 portions de ligne de $1 \mathrm{~m}$ de long. En même temps on note si la base de la fleur a été percée par les Bourdons à langue courte : (Bombus terrestris $\mathrm{L}$. et B. lucorum L.).

\section{$2^{\circ}-$ Les insectes pollinisateurs : étude du comportement et des spectres}

L'inventaire des espèces et le dénombrement des individus est effectué tous les jours, soit une fois le matin et une fois l'après-midi de chaque jour comme en 1972, soit une seule fois par jour, mais dans ce deuxième cas on fait alterner les comptages du matin et les comptages de l'après-midi, afin de ne pas introduire de biais systématique dans l'évaluation numérique globale. La surface de comptage est d'environ $200 \mathrm{~m}^{2}$ de végétation chaque fois $\left(100 \mathrm{~m}^{2}\right.$ sur les plantes mâle-fertile et autant sur les plantes mâle-stérile). On délimite une fois pour toutes 8 portions de 2 lignes adjacentes en bordure de bande, de $25 \mathrm{~m}$ de long (4 sur les mâle-fertile et 4 sur les mâle-stérile). Les portions de comptage sont réparties selon la diagonale des champs a fin d'avoir un échantillonnage représentatif de l'ensemble du champ (Fig. 2). Le comptage se fait en marchant lentement dans l'intervalle entre bandes, et en notant pour chaque individu rencontré, l'espèce, la caste, la présence de pelotes de pollen sur les pattes postérieures et enfin le lieu d'introduction de la langue. Une visite effectuée par l'entrée naturelle de la corolle est notée comme " positive " $\left({ }^{+}\right)$tandis qu'une visite effectuée par les trous percés à la base d'une corolle est notée comme négative (-).

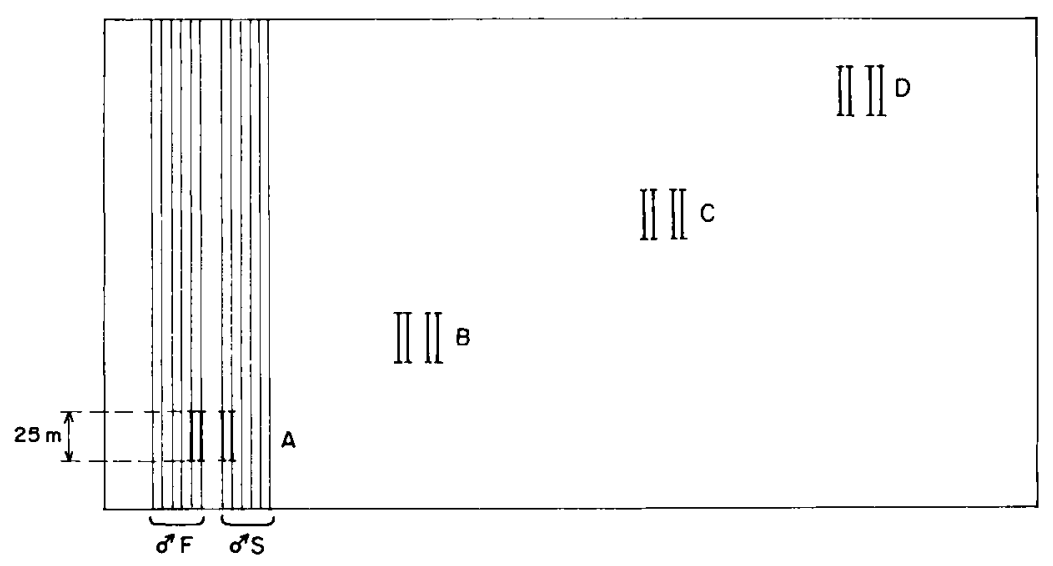

FIc. 2. - Emplacements des portions de lignes utilisées pour les comptages d'insectes sur un champ de féverole.

A, $\mathrm{B}, \mathrm{C}, \mathrm{D}=4$ emplacements de comptage.

$\because \mathrm{F}=$ bande de plantes mâle-fertile (6 rangs).

is $\mathrm{S} \quad=$ bande de plantes mâle-stérile ( 6 rangs).

Aвв. 2. - Die für die Insektenzählung im Bohnenfeld benutzten Stellen

A, B, C, D - 4 Zählstellen

A $\mathbf{F}=$ Streifen mit männlichen-fertilen Pflanzen (6 Reihen)

; $\mathrm{S} \quad=$ Streifen mit männlichen-sterilen Pflanzen (6 Reihen) 
Une étude plus fine du comportement de butinage est entreprise sur des individus pris au hasard dans le champ. L'opération consiste à suivre aussi longtemps que possible un insecte en chronométrant ses allées et venues et en notant le nombre de fleurs visitées, le type de visite (positive ou négative) et la lignée dans laquelle se trouve la plante butinée.

\section{$3^{0}$ - Étude particulière de l'abeille domestique}

Pendant les trois années consécutives le transport de ruches et l'emploi de nourrisseurs et de trappes à pollen (à trous de $5-6 \mathrm{~mm}$ ) a permis d'étudier la récolte du pollen par les ouvrières et l'effet du nourrissage avec un sirop de sucre à 50 pour cent sur cette récolte. L'élevage d'abeilles à Lusignan est composé en grande partie d'individus de race noire (Apis mellifica mellifica). Les ruches sont amenées dans les champs au début de la floraison de la féverole. Les trappes sont mises en service 2 jours par semaine. En 1972, on fait 2 prélèvements par jour de fonctionnement, l'un à midi, correspondant au butinage de la matinée et l'autre après l'arrêt d'activité, correspondant au butinage de l'après-midi. En 1973 et 1974 on se contente de prélever un échantillon représentatif de la récolte totale de la journée. Après chaque fonctionnement des trappes, qui sont au nombre de 4 , on mesure le volume total des pelotes et on analyse un échantillon de 3 à 400 pelotes en 2 étapes : la première est un tri par la couleur et la seconde est un contrôle du tri grâce aux techniques microscopiques bien connues en mélissopalynologie (cf. : Maurizio et Louveaux, 1965).

Quant au nourrissage il intervient en 1973 et 1974 a fin d'expérimenter une méthode préconisée par Free (1965) pour augmenter le nombre de butineuses de pollen. Chaque année 2 des 4 ruches sont nourries du début jusqu'à la mi-floraison puis on retire les nourrisseurs, tandis que les 2 autres ne sont pas nourries pendant la première phase de la floraison et sont nourries pendant la deuxième phase.

\section{$4^{0}$ - Étude de l'influence de la largeur de bandes mâle-stérile sur la pollinisation et la production de semence}

Sur chacun des champs expérimentaux où sont testés les 3 largeurs de bandes mâlestérile on fait une évaluation de la nouaison sur les lignes de bordure et sur les lignes médianes des bandes mâle-stérile dans les 3 dispositifs de culture de largeurs respectives : $2,5 \mathrm{~m} ; 5,0 \mathrm{~m}$; $7,5 \mathrm{~m}$. L'unité de comptage est 60 tiges soit 4 répétitions de 15 tiges.

A la maturité des gousses on fait la récolte de façon individuelle a fin de connaître le rendement de chaque bande mâle-stérile ou mâle-fertile.

\section{RÉSULTATS}

\section{A. - L'inventaire de la faune pollinisatrice}

Le recensement des apoïdes visitant la féverole dans 19 champs montre en 1973 (tableau 2) l'importance numérique de l'abeille domestique ( $79 \%$ des effectifs) et la modeste densité de la faune sauvage : bourdons et abeilles solitaires : 500 individus par jour et par hectare en moyenne, soit environ $20 \%$ des effectifs. Les bourdons sont 3 fois plus nombreux que les abeilles solitaires. Bien que certains doutes subsistent dans les déterminations faites à vue par les observateurs je donne sur la figure 3 le spectre moyen des espèces sauvages qui est composé par ordre d'abondance décroissante de : Bombus terrestris L. + B. lucorum L., Eucera tuberculata, B. hortorum L. + B. ruderatus Fab., B. lapidarius L., Anthophora acervorum L., Andrena ovatula K., B. sylvarum L., B. agrorum F., B. pratorum L. Suivant le champ, les espèces B. terrestris et $B$. lucorum, bourdons à langue courte, perceurs de corolles représentent de 6 à $74 \%$ de la faune sauvage (en moyenne $40 \%$ ). 
TABL. 2. - Inventaire de la faune pollinisatrice de féverole dans 8 départements français en 1973. TABL. 2. -Verzeichnis der die Pferdebohne in 8 französischen Departements bestäubenden Insekten. 1973

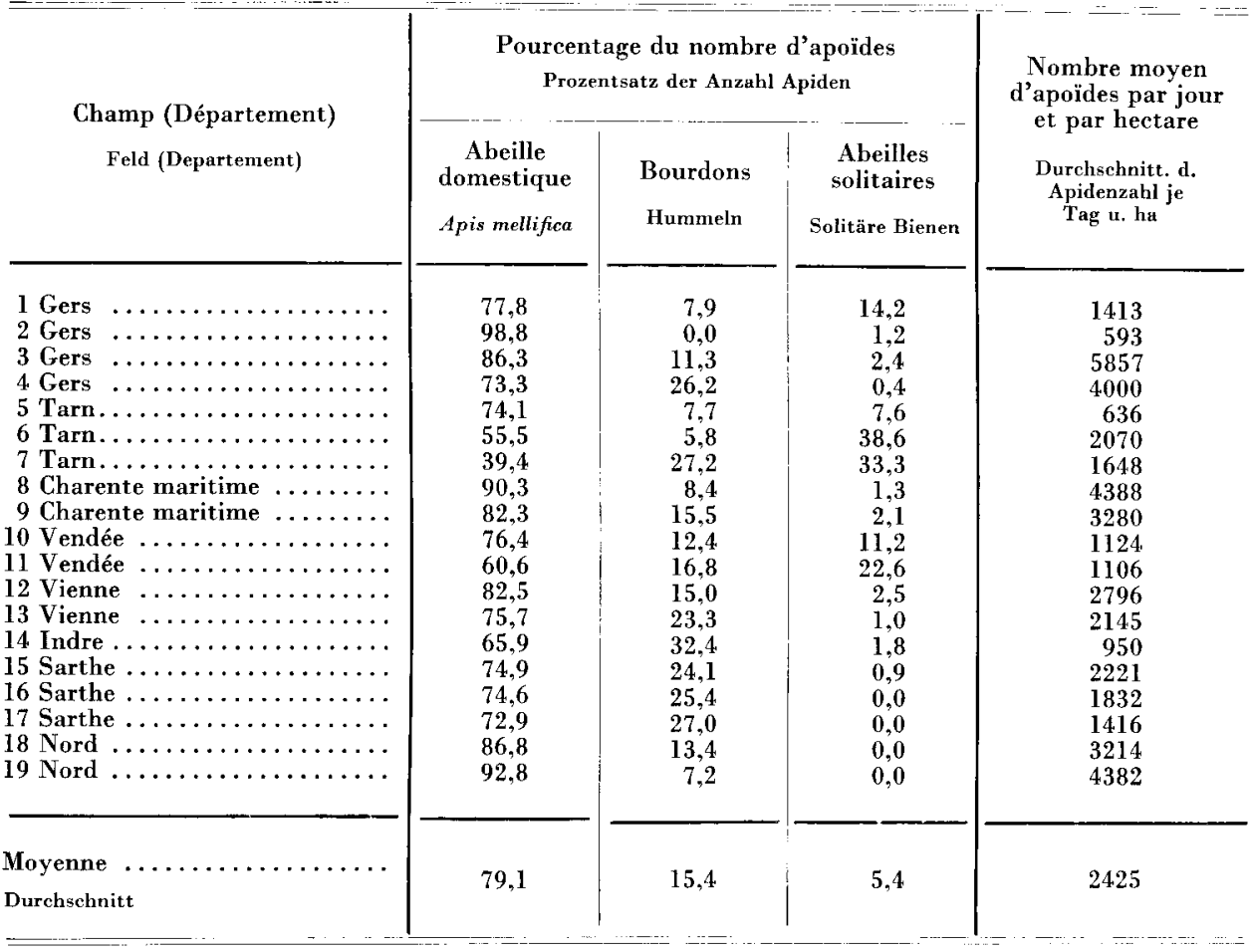

A Lusignan on possède un inventaire d'un autre intérêt puisqu'au même endroit, pendant 4 années consécutives on suit l'évolution des populations d'insectes. Le résultat global de l'inventaire se trouve dans le tableau 3 et les détails du spectre de la faune sauvage sur la figure 4 . On voit que l'abeille domestique est toujours relativement abondante (70 à $92 \%$ de la faune totale) et que la densité des bourdons est très variable d'une année à l'autre (262 à 1087 individus par hectare et par jour) de même que celles des abeilles solitaires (15 à 402 individus par hectare et par jour). Elle semble d'ailleurs liée à la superficie de féverole : la plus forte densité de bourdons s'observe sur le champ de 0,8 ha, la plus faible sur le champ de 6,5 ha, les densités intermédiaires correspondant aux superficies intermédiaires.

La prédominance des Terrestribombus (B. terrestris et $B$. lucorum) est constante, les espèces secondaires étant : $B$. lapidarius, $B$. hortorum et $B$. ruderatus. Quant aux abeilles solitaires c'est toujours Eucera tuberculata qui est la 


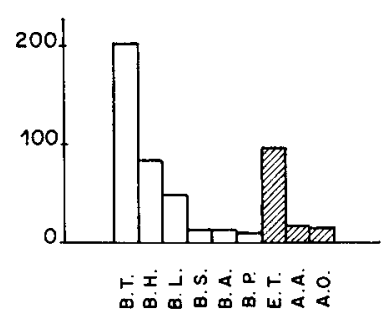

Fig. 3. - Spectre moyen des insectes pollinisateurs sauvages recensés dans 19 champs de féverole dans 8 départements français en 1973.

Verticalement $=$ nombre d'individus par jour et par hectare.

Авв, 3.-Durchschnittliches Spektrum der bestäubenden wilden Insekten,

die 1973 auf 19 Vicia faba Feldern in 18 französischen Departements festgestellt wurden.

Ordinate $=$ Anzahl Tiere je Tag und ha

$\square$ Bombus

QIITA Apoïdea solitaires

B.T. Terrestribombus

B.H. Hortobombus

B.I. Bombus lapidarius

B.S. Bombus sylvarum

B.A. Bombus agrorum.

B.P. Bombus pratorum

E.T. Eucera tuberculata

A.A. Anthophora acervorum

A.O. Andrena ovatula

TABL. 3. - Inventaire global de la faune pollinisatrice de la féverole à Lusignan.

TAB. 3. - Gesamtverzeichnis der bestäubenden Insekten in Lusignan.

\begin{tabular}{|c|c|c|c|c|}
\hline \multirow{2}{*}{$\begin{array}{l}\text { Année } \\
\text { Jahr }\end{array}$} & \multirow{2}{*}{$\begin{array}{c}\text { Nombre de } \\
\text { comptages } \\
\text { sur } 200 \mathrm{~m}^{2} \\
\text { Zählungen auf } 200 \mathrm{~m}^{2}\end{array}$} & \multicolumn{3}{|c|}{$\begin{array}{l}\text { Nombre moyen d'individus recensés par jour } \\
\text { et par hectare } \\
\text { Durehschnittliche Zahl von Individuen festgestellt je Tag u. ha }\end{array}$} \\
\hline & & $\begin{array}{c}\text { Abeille domestique } \\
\text { Apis mellifica }\end{array}$ & $\begin{array}{l}\text { Bourdons } \\
\text { Hummeln }\end{array}$ & $\begin{array}{c}\text { Abeilles solitaires } \\
\text { Solitäre Bienen }\end{array}$ \\
\hline $\begin{array}{l}1971 \\
1972 \\
1973 \\
1974\end{array}$ & $\begin{array}{l}17 \\
33 \\
20 \\
21\end{array}$ & $\begin{array}{l}1564,(70,2) \\
1195(79,6) \\
6328(91,9)\end{array}$ & $\begin{array}{l}1087 \\
646(29,0) \\
262(17,4) \\
540(7,8)\end{array}$ & $\begin{array}{l}402 \\
18(0,8) \\
45(3,0) \\
15(0,2)\end{array}$ \\
\hline
\end{tabular}

( ) - Pourcentage du nombre total des apoïdes.

( ) $=\%$ der Gesamtzahl an Apiden 


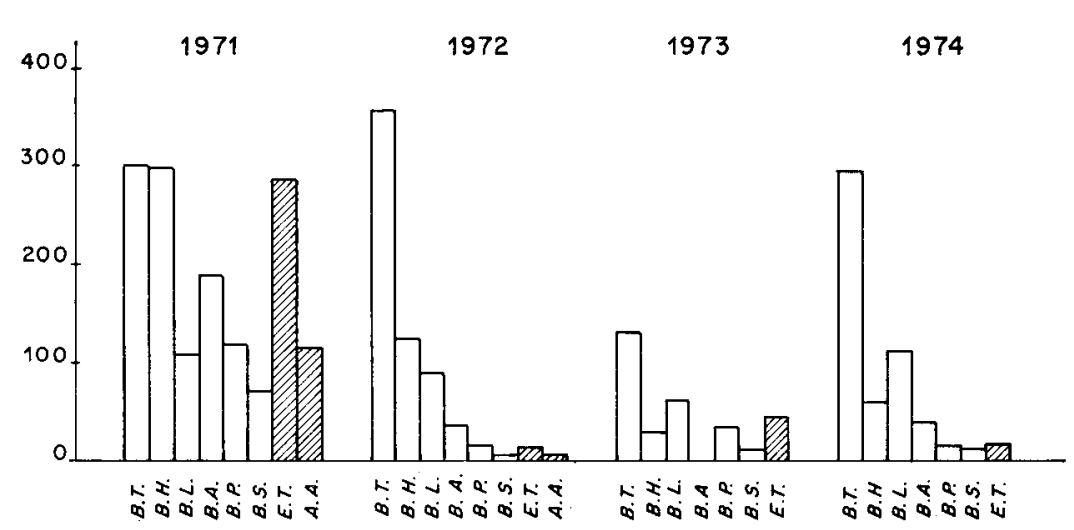

FIg. 4. - Spectres annuels de la faune pollinisatrice de la féverole à Lusignan. Même légende que Fig. 3.

Aвв. 4. - Jahresspektrum der Vicia faba bestäubenden Fauna in Lusignan. Legende wie in Abb. 3.

mieux représentée et parfois la seule (fig. 5). Ces résultats sont assez semblables à ceux de l'enquête effectuée en 1973 dans plusieurs régions de France.

Au total on a pu recenser 14 espèces d'abeilles sauvages : B. terrestris L., B. lucorum L., B. lapidarius L., B. hortorum L., B. ruderatus Fab., B. agrorum F., B. sylvarum L., B. pratorum L., B. equester Fab., B. hypnorum L. (ces deux dernières espèces étant très rares), Eucera tuberculata F., Anthophora acervorum L., Andrena ovatula K., et Andrena flavipes Panz. (très rares exemplaires).

La distinction des castes lors des comptages (tableau 4) permet de situer la synchronisation de la floraison à Lusignan et du cycle de développement des colonies de bourdons. On peut dire que seuls les Hortobombus (B. hortorum et $B$. ruderatus) sont en tout début de cycle puisque seules les reines butinent, alors que $B$. agrorum, $B$. pratorum et les Terrestribombus sont en pleine production d'ouvrières. B. lapidarius et $B$. sylvarum sont des espèces à précocité intermédiaire.

\section{B. L Le comportement de butinage des espèces}

1. Le percement des corolles et les visites « positives 》 et « négatives 》

Deux espèces à langue courte $B$. terrestris et $B$. lucorum percent la base des corolles (fig. 5 e). Leur travail est assez important puisque 50 à $100 \%$ des fleurs sont percées tout au long de la floraison (fig. 6). Reines et ouvrières sont 

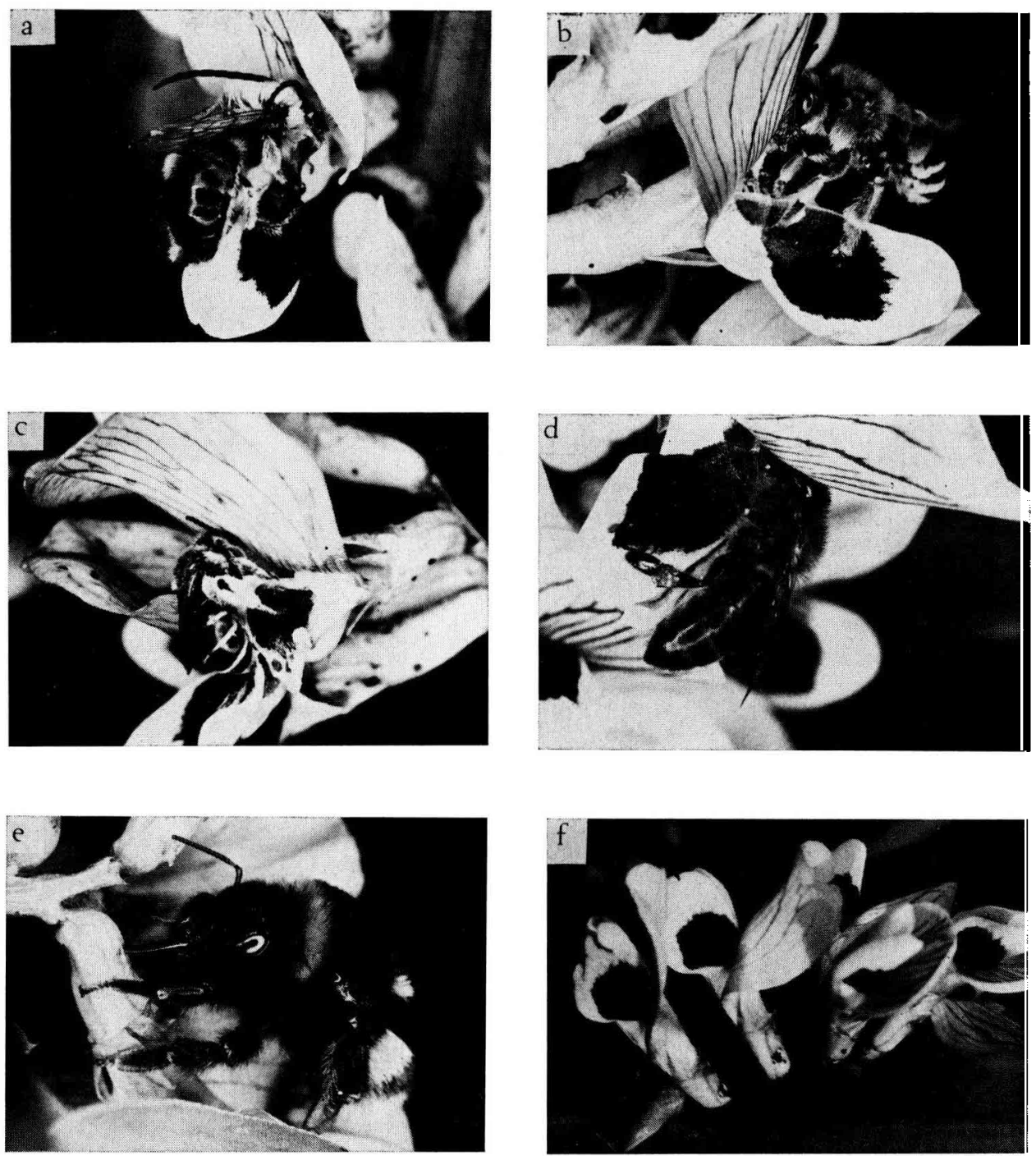

Fig. 5. - Apoidea de la féverole (Photo S. Carre).

a) Mâle d'Eucera tuberculata

b) Femelle d'Eucera tuberculata

c) Femelle d'Andrena ovatula

d) Abeille domestique " positive "

e) Reine de Bombus terrestris

f) Fleur de féverole à calice percé par Bombus terrestris.

Aвв. 5. -- Vicia faba besuchende Apiden (S. Carre phot).

a) Männchen von Eucera tuberculata

b) Weibchen von Eucera tuberculata

c) Weibchen von Andrena ovatula

d) «positive » Apis mellifica

e) Königin von Bombus terrestris

f) Pferdebohnenblïte mit einem von Bombus terrestris durchgebohrten Blumenkelch. 
TABL. 4. - Abondance relative des reines et des ouvrières de quelques espèces de Bourdons pendant la floraison de la féverole à Lusignan. TAв. 4. 一 Relative Häufigkeit von Königinnen und Arbeiterinnen einiger Hummelarten während der Blütezeit von Vicia faba equina in Lusignan.

\begin{tabular}{|c|c|c|c|}
\hline \multirow[b]{2}{*}{$\begin{array}{c}\text { Espèce } \\
\text { Art }\end{array}$} & \multicolumn{3}{|c|}{$\begin{array}{l}\text { Nombre d'individus } \\
\text { recensés au cours des } 3 \text { ans } \\
\text { Anzahl d. Tiere, die im Verlauf von drei } \\
\text { Jahren festgestellt wurden }\end{array}$} \\
\hline & $\begin{array}{c}\text { Reines } \\
\text { Königinnen }\end{array}$ & $\begin{array}{l}\text { Ouvrières } \\
\text { Arbeiterinnen }\end{array}$ & $\begin{array}{c}\text { Pourcentage } \\
\text { d'ouvrières } \\
\% \text { der Arbeite- } \\
\text { rinuen }\end{array}$ \\
\hline 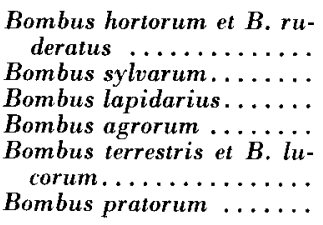 & $\begin{array}{r}110 \\
11 \\
135 \\
25 \\
\\
248 \\
11\end{array}$ & $\begin{array}{r}7 \\
1 \\
13 \\
13 \\
167 \\
\\
27\end{array}$ & $\begin{array}{r}5,9 \\
8,3 \\
8,8 \\
34,2 \\
40,2 \\
71,0\end{array}$ \\
\hline
\end{tabular}

toutes les deux capables de percer calice et corolle ou d'utiliser les trous faits par d'autres individus, adoptant ainsi un comportement « négatif ». Les autres apoïdes à langue courte (6 à $12 \mathrm{~mm})$ tels que l'abeille domestique, $B$. pratorum, B. sylvarum, B. agrorum, B. lapidarius ne percent pas les fleurs mais utilisent plus ou moins fréquemment les trous de Terrestribombus. Seuls les Hortobombus à langue longue $(16 \mathrm{~mm})$ ne se laissent jamais tenter par les morsures à la base des fleurs et ils s'introduisent dans les corolles par l'entrée naturelle. Ce sont des bourdons toujours « positifs ». Il en est de même pour les abeilles solitaires : Eucera et Anthophora. Le tableau 5 permet de classer en 3 groupes les apoïdes :

- ceux qui visitent toujours positivement les fleurs de féverole : Hortobombus et abeilles solitaires,

- ceux qui visitent les fleurs le plus souvent de façon positive : $B$. lapidarius, B. agrorum, B. sylvarum,

- ceux qui visitent les fleurs le plus souvent de façon négative : $B$. pratorum, Terrestribombus, abeille domestique.

Les figures 7, 8 et 9 illustrent le comportement de récolte selon l'année, le jour, l'espèce de bourdon, la caste et la lignée de féverole. Chez les Terrestribombus, reines et ouvrières peuvent avoir un comportement " positif », mais ceci surtout en début de floraison. De même les reines et les ouvrières de B. lapidarius à comportement négatif ne se rencontrent que dans la deuxième moitié de la floraison. L'examen des figures 10,11 et 12 conduit à une constata- 


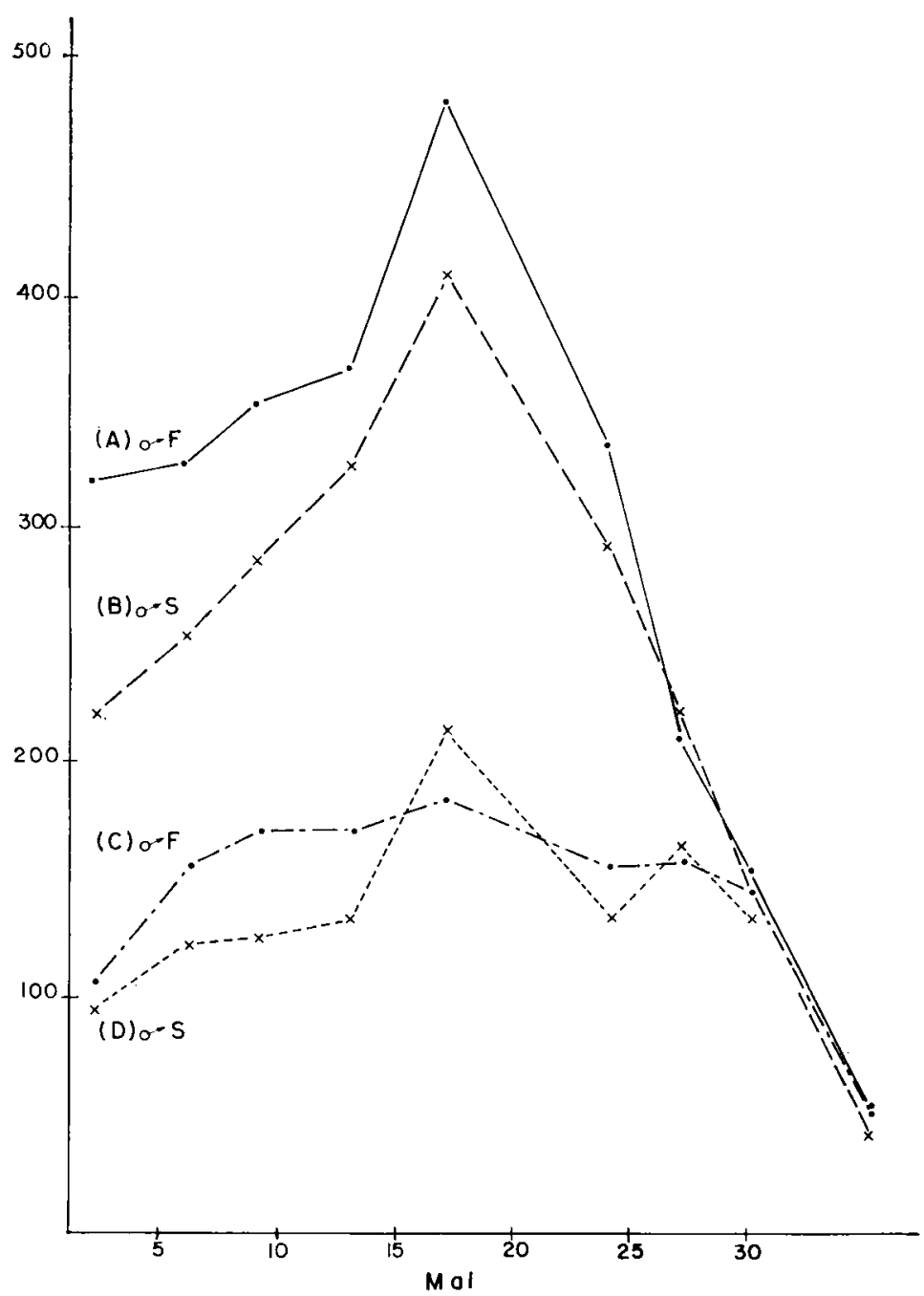

Fig, 6. - Floraison et percement des corolles par Terrestribombus sur les plantes mâle-fertile et mâle-stérile de féverole à Lusignan 1974.

Verticalement $=$ Nombre de fleurs par mètre linéaire.

Horizontalement $=$ Date.
$A, B=$ fleurs épanouies
$\mathrm{C}, \mathrm{D}=$ fleurs percées
3 $\mathbf{F}=$ plantes mâle-fertile
‡ $\mathbf{S}=$ plantes mâle-stérile

Авв. 6. - Blütezeit und Durchlöchern der Blumenkronen durch Bombus terrestris auf männlich-fertilen und männlich-sterilen Pflanzen von Vicia faba in Lusignan 1974.

Ordinate - Zahl der Blüten je Meter

Abszisse $=:$ Datum

A, $\mathbf{B}-$ verwelkte Bliiten

$\mathrm{C}, \mathrm{D}=$ durchlöcherte Bläten

$\overrightarrow{5} \mathbf{F}=$ männlich-fertil

‡ $\mathbf{S}=-$ männlich-steril 
TABL. 5. - Tendance à visiter " positivement » les fleurs de féverole chez les apoïdes recensés au cours des inventaires à Lusignan.

Tав. 5. - Tendenz der im Verlauf der Untersuchungen in Lusignan festgestellten Apiden, die Blüten der Pferdebohne " positiv » zu besuchen.

\begin{tabular}{|c|c|c|c|c|}
\hline \multirow[b]{2}{*}{ Espèce } & \multicolumn{4}{|c|}{$\begin{array}{l}\text { Pourcentage d'individus de chaque espèce } \\
\text { effectuant des visites « positives »" } \\
\text { Prozentsatz d. Individuen jeder Art, } \\
\text { die " positive "Besuche ausführten }\end{array}$} \\
\hline & 1972 & 1973 & 1974 & $\begin{array}{c}\text { Moyenne } \\
\text { Durchsehnitt }\end{array}$ \\
\hline 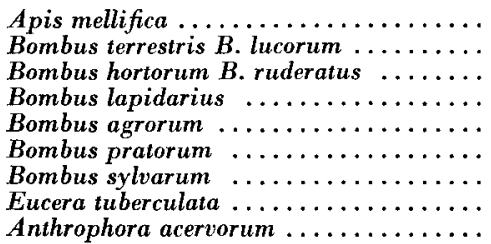 & $\begin{array}{l}17,3 \\
6,0 \\
100 \\
86,7 \\
100 \\
14,3 \\
75,0 \\
100 \\
100\end{array}$ & $\begin{array}{l}5,9 \\
11,5 \\
100 \\
92,0 \\
\frac{0}{50,0} \\
100 \\
-\end{array}$ & $\begin{array}{c}13,6 \\
12,2 \\
100 \\
74,0 \\
58,8 \\
71,4 \\
100 \\
100 \\
\end{array}$ & $\begin{array}{c}13,6 \\
9,9 \\
100 \\
84,2 \\
79,4 \\
28,6 \\
75,0 \\
100 \\
100\end{array}$ \\
\hline
\end{tabular}

1. Visites effectuées par l'entrée naturelle de la corolle par opposition aux visites « négatives " effectuées par les trous de la base de la carolle.

1. Besuch durch den natürlichen Eingang der Blumenkrone im Gegensatz zum « negativen» Besuch durch die Löcher an der Blumenkronen-Basis.

tion analogue chez l'abeille domestique : les individus ( négatifs ) sont surtout abondants après les 10 ou 15 premiers jours de floraison; le pourcentage d'ouvrières « positives m relativement élevé au début de la floraison, ne cesse de décroître jusqu’à la fin.

Les visites d'insectes sur les nectaires des stipules sont très peu fréquentes : en 1971 seule l'espèce solitaire et terricole Andrena carantonica Perez butine sur les stipules et jamais sur les fleurs. En 1973 quelques abeilles domestiques font des visites stipulaires mais seulement en fin de floraison.

\section{Les vitesses de butinage}

On voit dans le tableau 6 que les vitesses de butinage varient beaucoup d'une espèce à l'autre. Les plus rapides sont les Hortobombus et la plus lente est l'abeille domestique. La rapidité de visite est d'ailleurs fonction du type de butinage, par exemple chez l'abeille domestique les récolteuses de pollen (positives) sont plus lentes que les récolteuses de nectar (négatives) (tableau 7). 

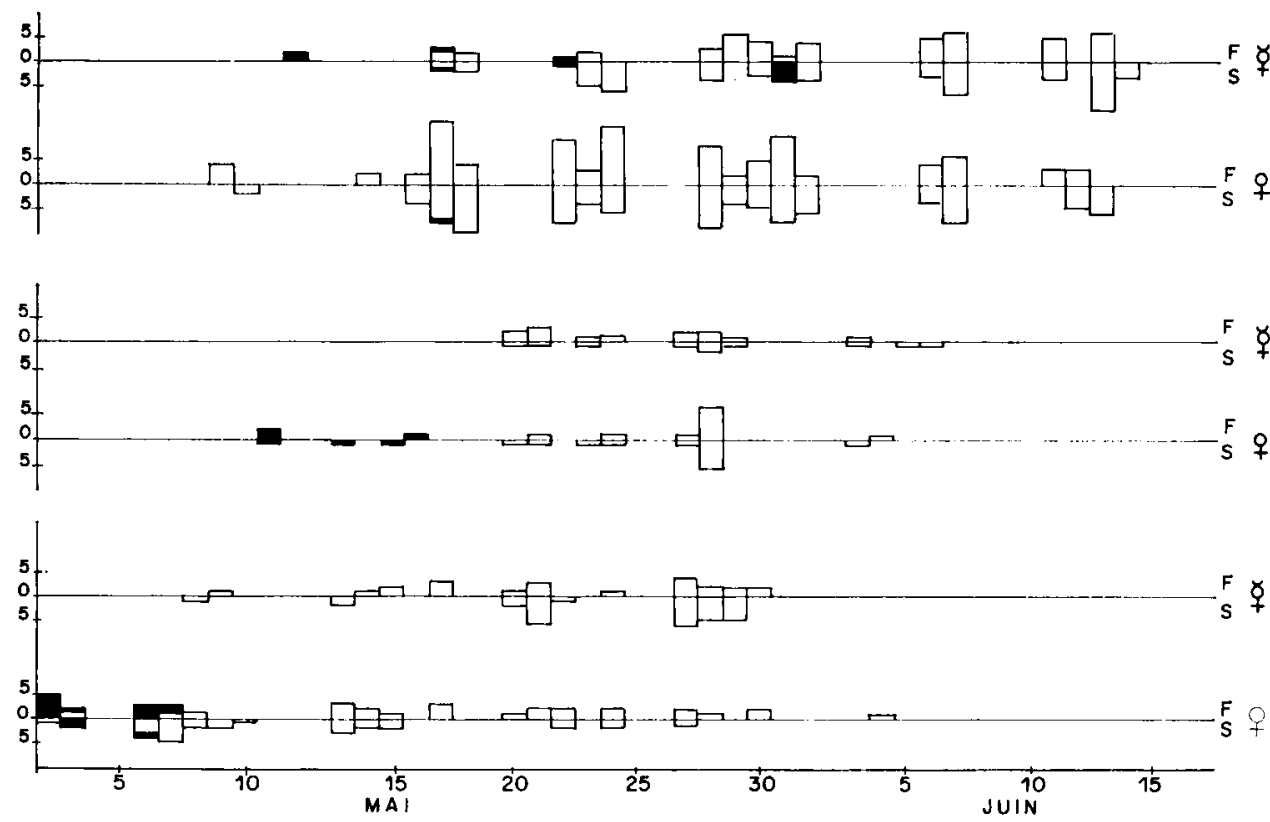

BOMBUS PRATORUM
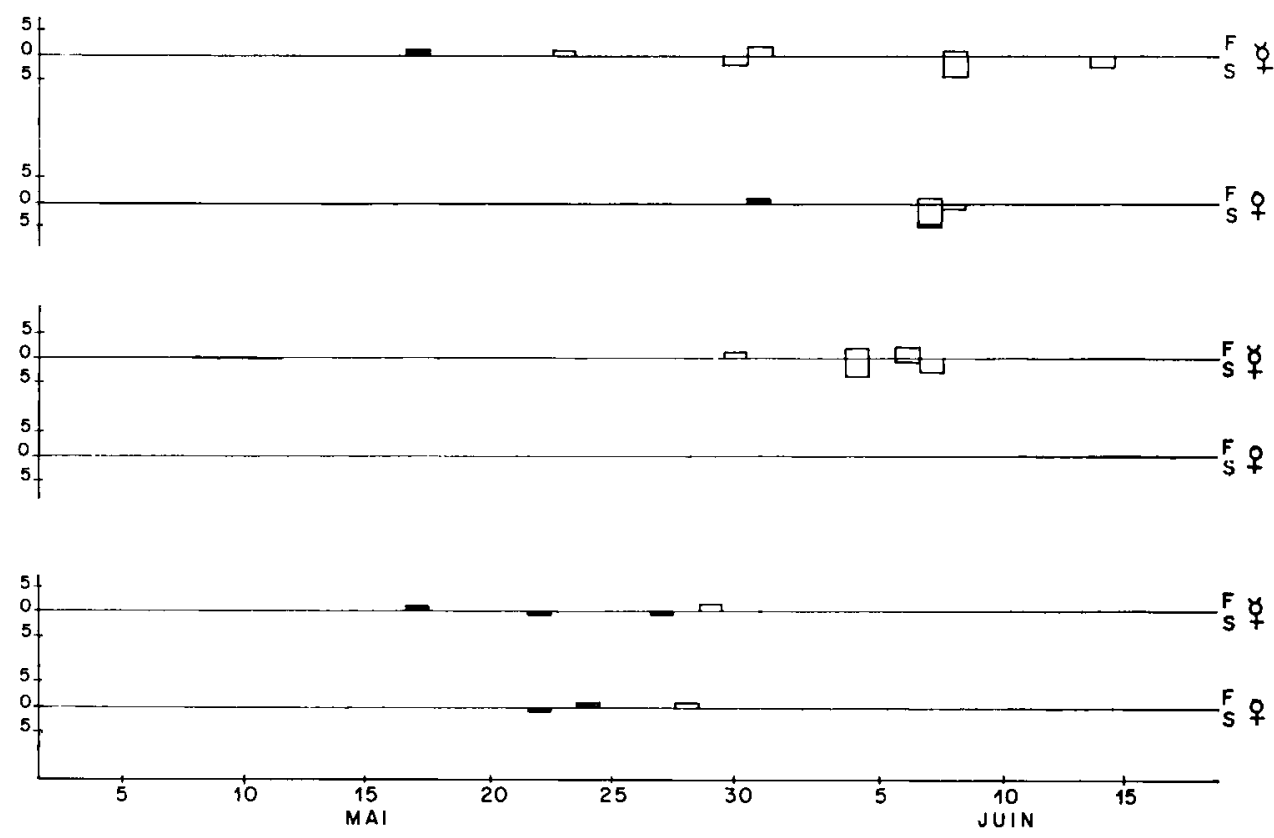

FIg. 7. - Inventaire des Bourdons sur les deux lignées de fëverole à Lusignan. Terrestribombus et Bombus pratorum.

Verticalement $=$ Nombre d'individus sur $100 \mathrm{~m}^{2}$. Horizontalement $=$ Date

$$
\begin{aligned}
& \square=\text { visite négative } \\
& \text { = visite positive }
\end{aligned}
$$

F - plantes mâle-fertile $\mathrm{S}=$ plantes mâle-stérile.
Ordinate $=$ Anzahl Tiere auf $100 \mathrm{~m}^{2}$ Abszisse = Datum

$$
\begin{aligned}
& =\text { negativer Besuch } \\
& =\text { positiver Besuch }
\end{aligned}
$$

$\mathrm{F}=$ männlich-fertile Pflanze

$S$ männlich-sterile Pffanze

Авв. 7. - Verzeichnis der Hummeln auf den beiden Stämmen der Pferdebohne in Lusignan. 


\section{BOMBUS LAPIOARIUS}
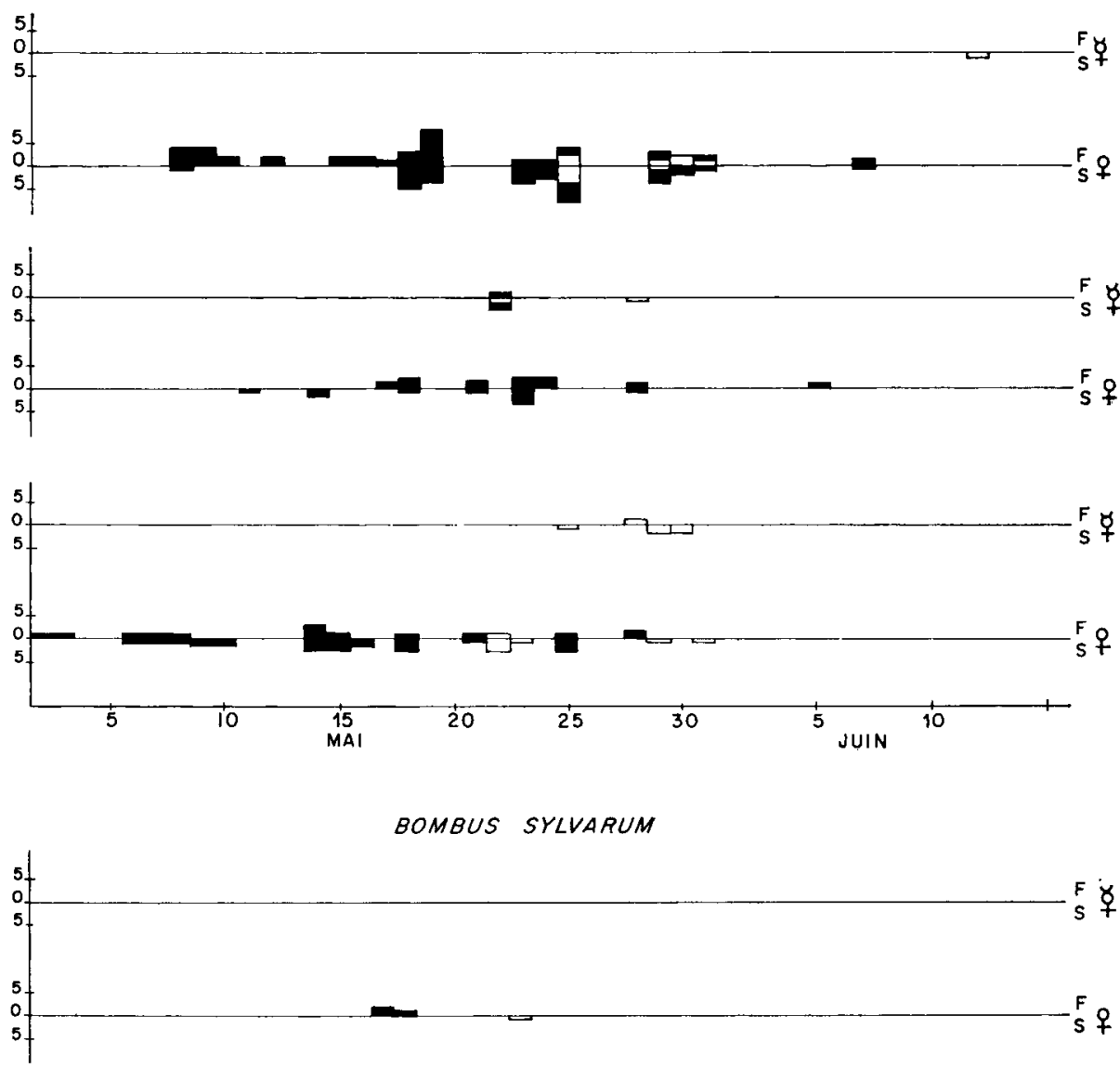

$$
\begin{aligned}
& 5 \\
& 0 \\
& 5 \\
& 5 \\
& 5 \\
& 0 \\
& 5
\end{aligned}
$$
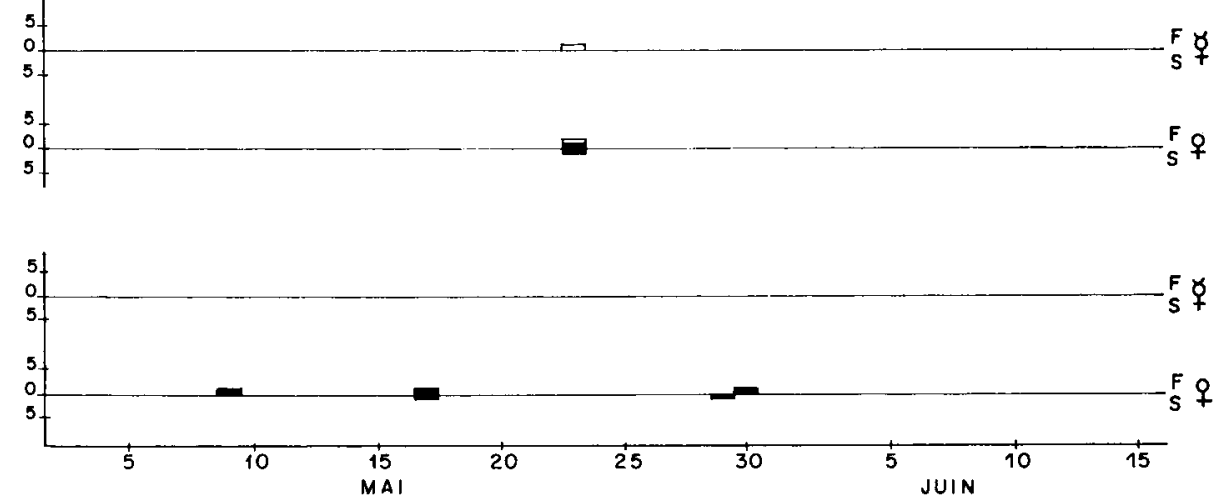

Fig. 8. - Inventaire des Bourdons sur les deux lignées de féverole à Lusignan. Même légende que la Fig. 7.

Bombus lapidarius et Bombus sylvarum.

Aвв. 8. - Verzeichnis der Hummeln auf den beiden Stämmen der Pferdebohne in Lusignan. Legende wie in Abb. 7 . Bombus lapidarius, Bombus sylvarum. 


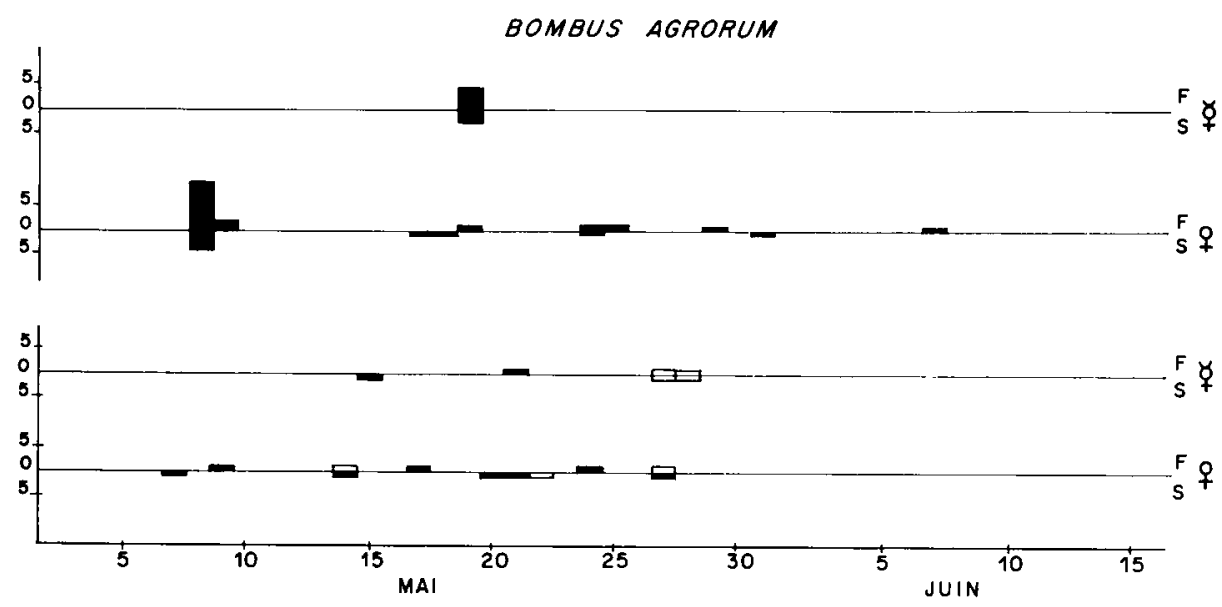

1972 s 우
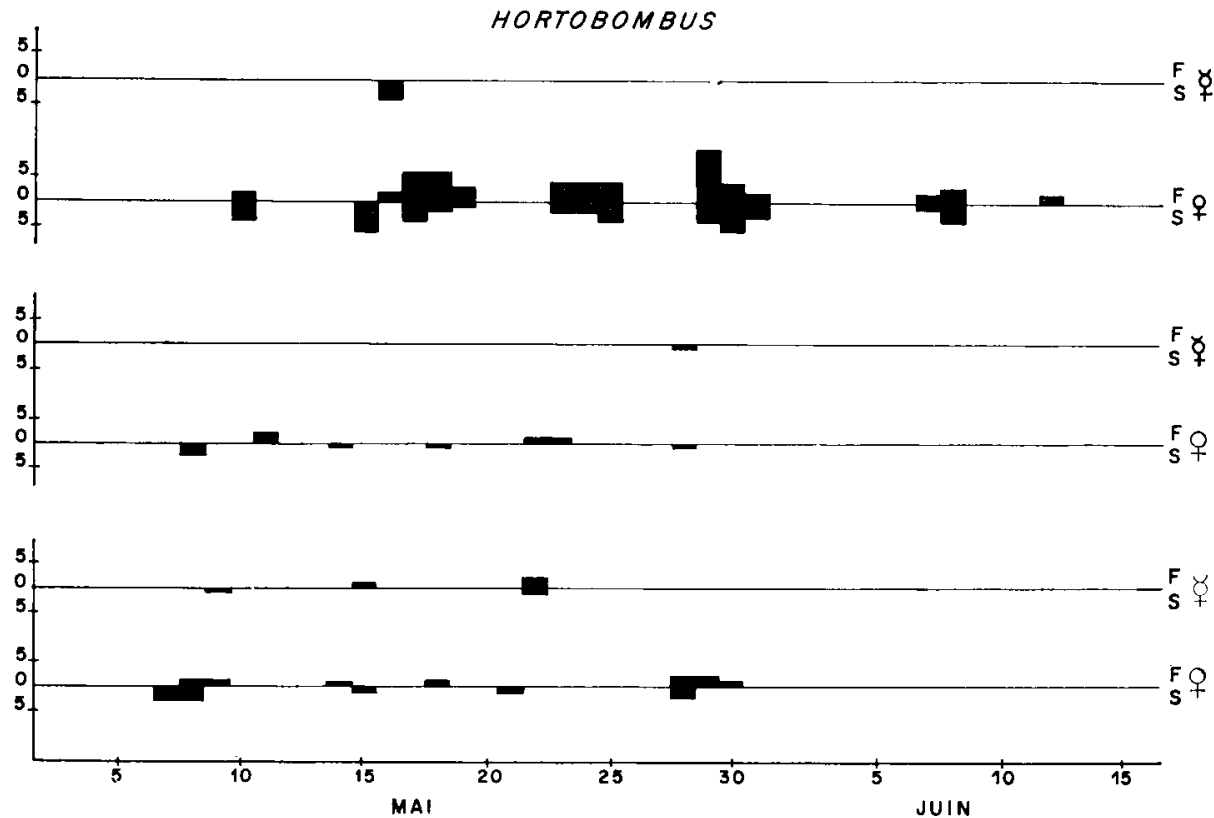

Fig. 9. - Inventaire des Bourdons sur les deux lignées de féverole à Lusignan. Bombus agrorum et Hortobombus.

Même légende que la Fig. 7 .

Aв8. 9. -- Verzeichnis der Hummeln auf den beiden Stämmen der Pferdebohne in Lusignan. Legende wie in Abb. 7. 


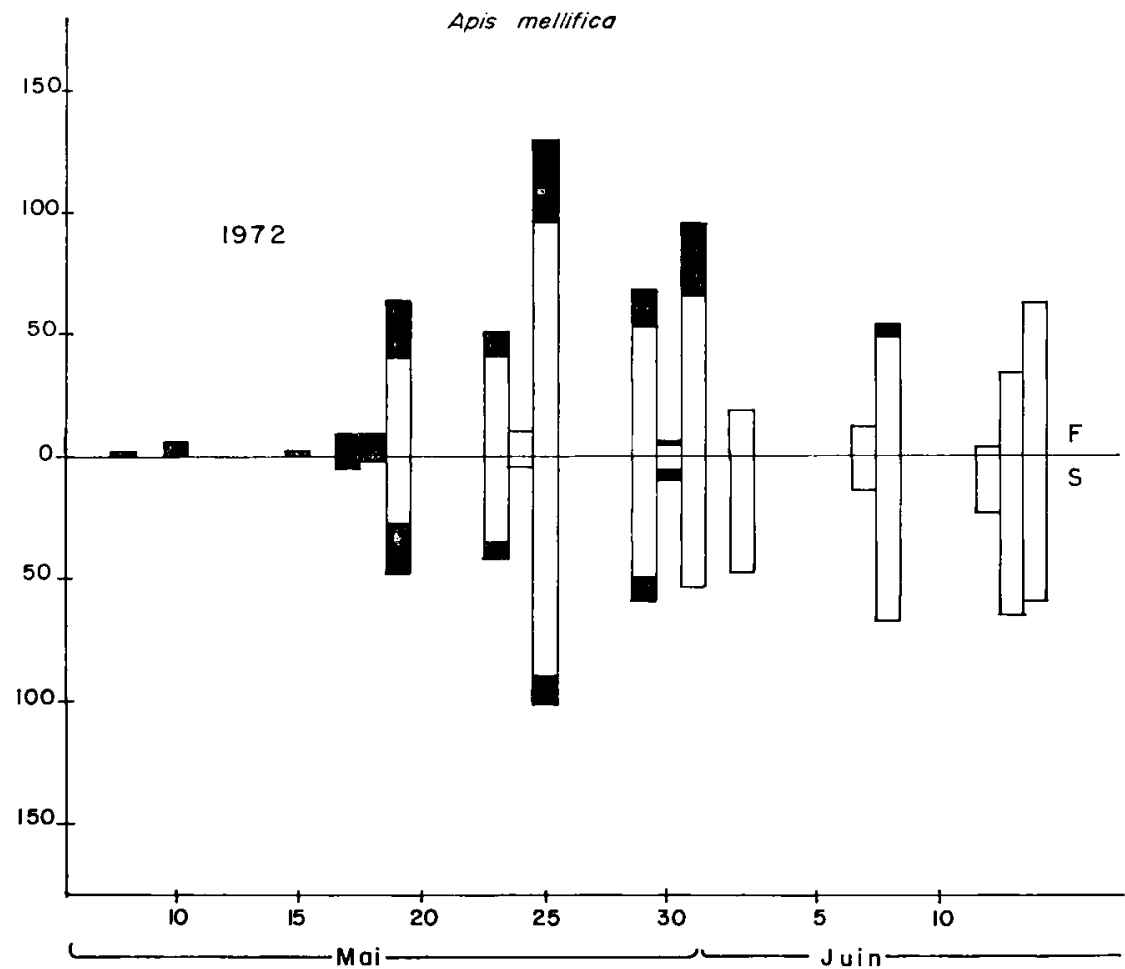

FIG. 10. - Inventaire des abeilles domestiques sur les deux lignées de fếverole à Lusignan en 1972. Même légende que la Fig. 7.

Авв. 10. - Verzeichnis von Apis mellifica auf den beiden Stämmen der Pferdebohne in Lusignan 1972. I.egende wie in Abb. 7 .
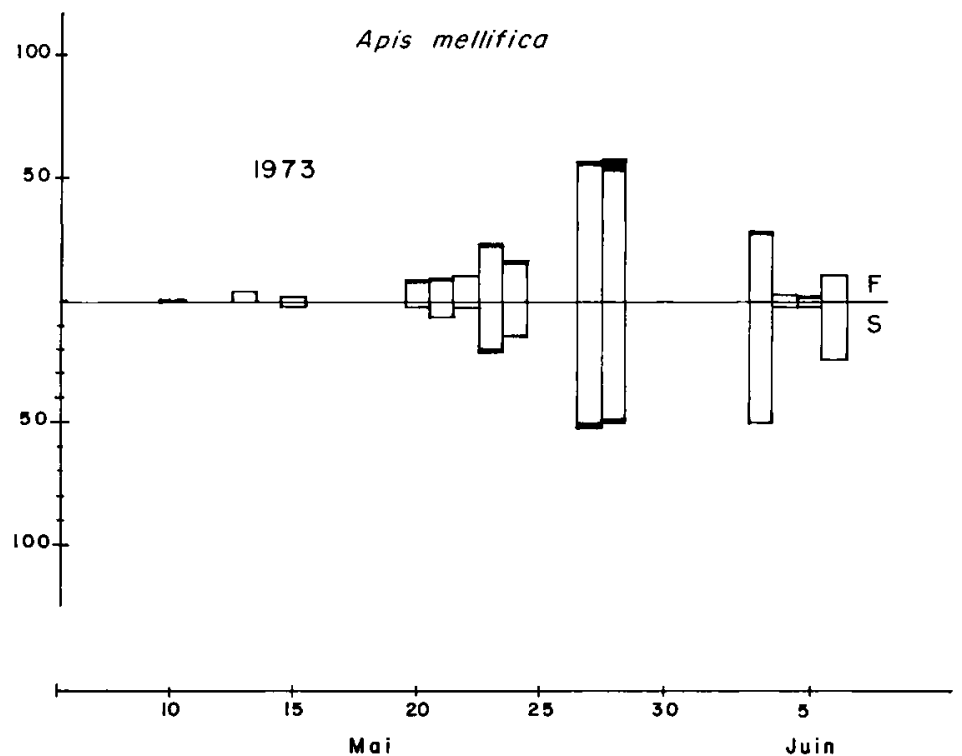

F1G. 11. - Inventaire des abeilles domestiques sur les deux lignées de féverole à Lusignan en 1973. Même légende que la Fig. 7.

Aвв. 11. - Verzeichnis von Apis mellifica auf den beiden Stämmen von Vicia faba in Lusignan 1973. Legende wie in Abb. 7. 


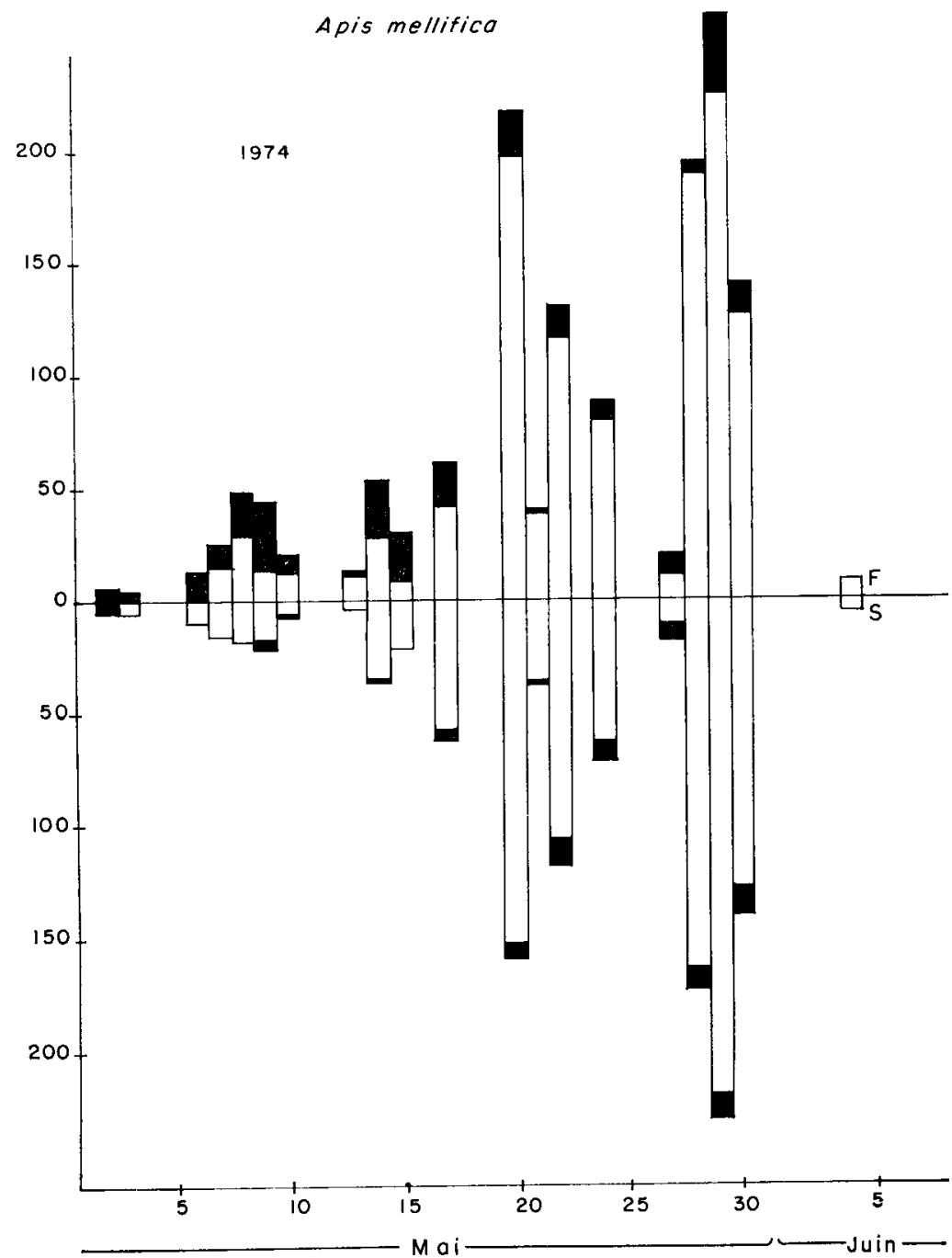

Fig. 12. - Inventaire des abeilles domestiques sur les deux lignées de féverole à Lusignan en 1974. Même légende que la Fig. 7.

Aв8. 12. - Verzeichnis von Apis mellifica auf den beiden Stämmen der Pferdebohne in Lusignan 1974. Legende wie in Abb. 7 
TABL. 6. --Vitesse de butinage moyenne des apoïdes rencontrés sur la féverole à Lusignan (1972-73-74).

Tав. 6. - Durchschnittsgeschwindigkeit der Apiden beim Sammeln auf Vicia faba; Lusignan 1972-73-74.

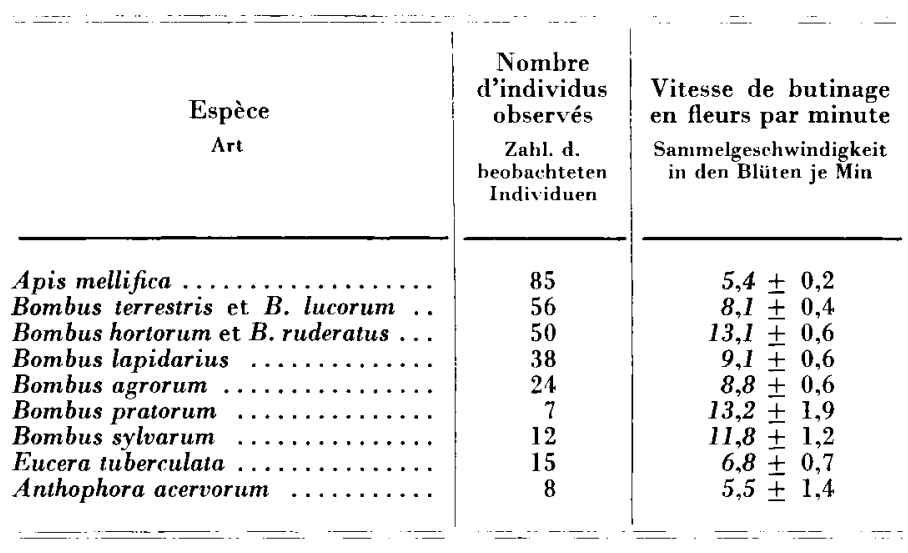

TABL. 7. -Vitesse de butinage de l'Abeille domestique selon son comportement à l'égard des fleurs de fêverole à Lusignan.

TAB. 7. - Sammelgeschwindigkeit von Apis mellifica nach ihrem Verhalten der Pferdebohne gegenüber; Lusignan.

Type de comportement vis-à-vis des fleurs. Typus des Verhaltens d. Blüten gegeniüber

Nombre d'ouvrières observées.

Zahl d. beobachteten Arbeiterinnen

Vitesse de butinage moyenne (Nombre de fleurs par minute)

Durchschnittliche Sammelgescliwindigkeit (7idt. Blüten je Min)

1. $+=$ visite positive (cf. tableau 5); positiver Besuch (S. Tab. 5).

2 . - visite négative; negativer Besuch. 


\section{L'activité de butinage selon le moment de la journée}

Le tableau 8 fait apparaître que l'abeille domestique fréquente la féverole 3 fois plus l'après-midi que le matin tandis que la population de bourdons ( positifs ) ou c négatifs » n'est pas significativement plus abondante l'aprèsmidi que le matin (tests de $\mathrm{F}$ à $5 \%$ ). Qu'il s'agisse du dénombrement des individus " positifs ", " négatifs ", butinant sur plantes mâle-fertile, ou sur plantes mâle-stérile, on constate que l'abeille domestique est dans tous les cas plus fréquente l'après-midi que le matin (tests de F significatifs à $1 \%$ ).

TABL. 8. - Abondance et comportement de l'Abeille domestique et des Bourdons en fonction du moment de la journée et de la fertilité des étamines. Lusignan 1972.

TAB. 8. - Häufigkeit und Verhalten von Apis mellifica und der Hummeln in Abhängigheit von Tageszeit und Reife der Staubgefässe. Lusignan 1972.

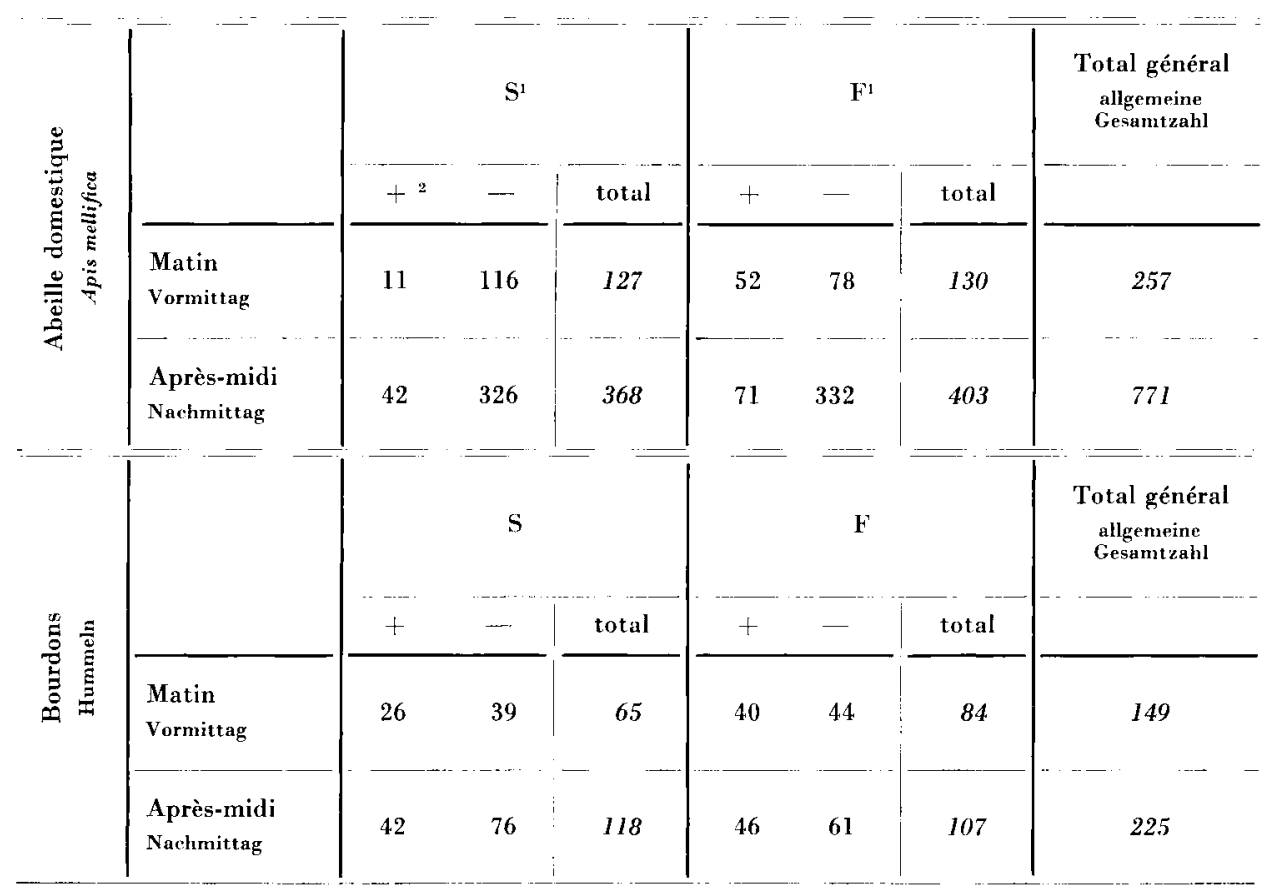

1. S plantes mâle-stérile , lignées isogéniques

F plantes mâle-fertile $j$ isogene Stämme.

2. + comportement " positif" 2. + = "positives "Verhalten. - comportement " négatif" _... ".. "negatives "Verhalten.

Chaque nombre correspond au nombre d'individus recensés au cours de 14 comptages effectués chacun sur environ $100 \mathrm{~m}^{2}$ de végétation.

Jede Zahl entspricht der Anzahl Tiere, die im Verlauf von 14 Zählungen ermittelt wurden. Jede Zählung erfolgte auf etwa $100 \mathrm{~m}^{2}$ bep flanzter Fläche. 


\section{La préférence pour l'une des lignées parentales}

Il est difficile de distinguer sur les figures 7 à 12 une attirance pour l'une ou l'autre des lignées chez l'une des espèces observées. L'analyse statistique des données ne peut être effectuée que de 1972 à 1974. Pendant cette période on ne décèle chez les abeilles sauvages aucune attirance significative pour l'une ou l'autre des lignées. Seule l'abeille domestique a une légère préférence pour les plantes mâle-fertile (test de F significatif à $10 \%$ ).

TABL. 9. - Attractivitê des lignêes de féverole pour les apoïdes à Lusignan.

TAB. 9. - Anziehungshraft der Vicia faba - Stämme auf die Apiden in Lusignan

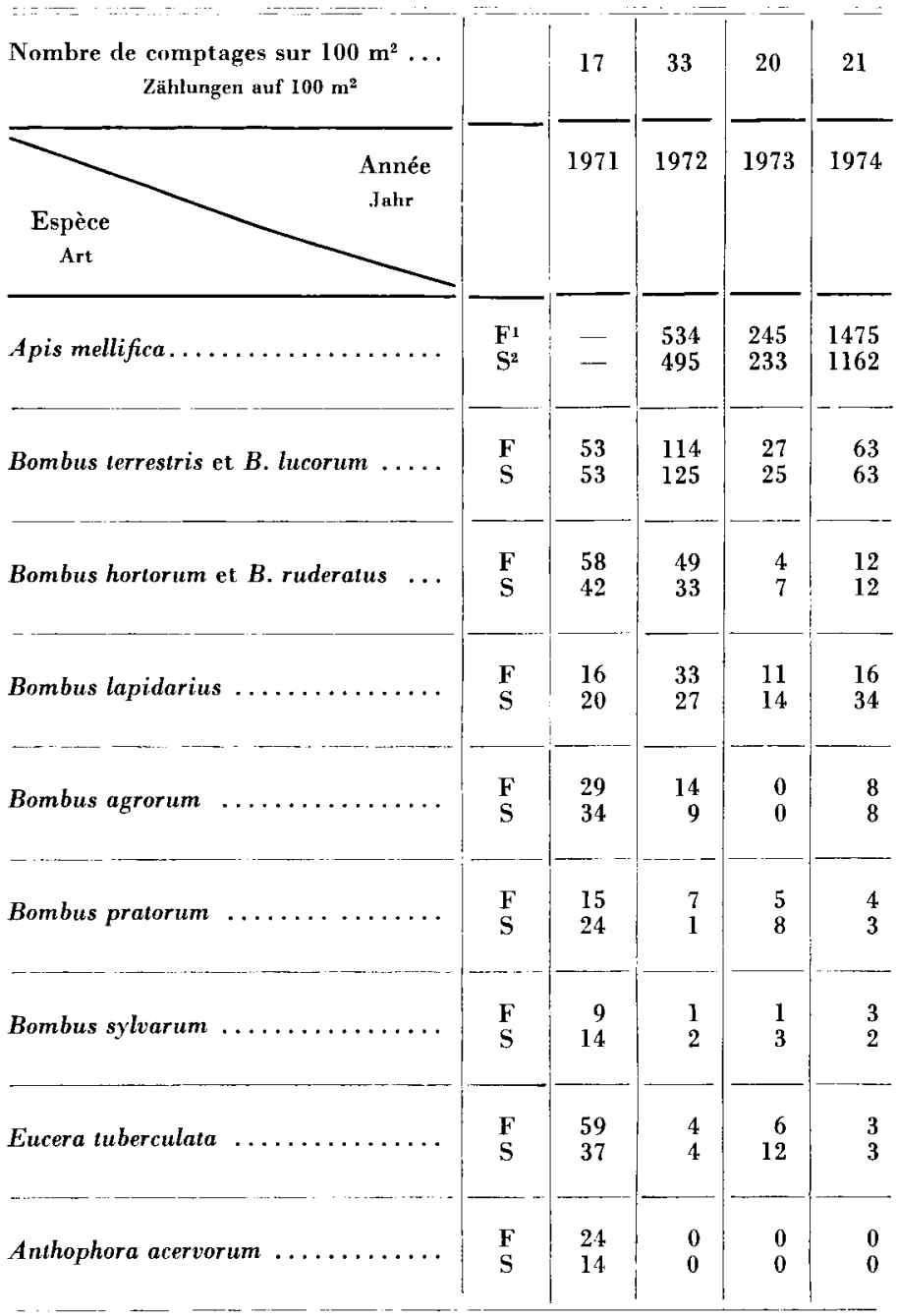

1. et 2, cf. : tableau 1. 1. und 2. *iehe Tubelle 1. 
Par contre si on considère les divers types de butineuses d'abeille domestique on se rend compte de l'influence très nette de la lignée (tableau 10) : les butineuses « négatives 》 se répartissent de façon à peu près équivalente sur les 2 lignées alors que les butineuses “ positives " sont près de 3 fois plus nombreuses sur les plantes mâle-fertile que sur les plantes mâle-stérile. Par ailleurs l'analyse des données de 1972 (tableau 8) montre que l'accroîssement du nombre de butineuses ( positives ) l'après-midi est plus fort sur les plantes mâle-fertile que sur les plantes mâle-stérile ( $F$ significatif à $5 \%$ ).

TABL. 10. - Abondance des abeilles domestiques à comportement « négatif " et à comportement « pasitif» sur les plantes mâle-fertile et mâle-stérile de fêverole à Lusignan pendant 3 ans.

Tав. 10. - Häufigkeit von Apis mellifica mit «negativem " und "positivem » Verhalten auf den männlich-fertilen und männlich-sterilen Pflanzen von Vicia faba während dreier Jahre in Lusignan.

\begin{tabular}{|c|c|c|c|c|c|}
\hline \multirow[t]{2}{*}{ 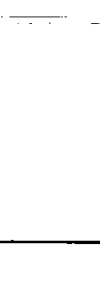 } & \multicolumn{4}{|c|}{$\begin{array}{l}\text { Pourcentage du nombre total d'abeilles } \\
\text { Prozentsatz der Gesamtzahl an Bienen }\end{array}$} & $\begin{array}{l}\text { Nombre total } \\
\text { d'individus } \\
\text { recensés } \\
\text { Gesantatahl der } \\
\text { fostgestellten } \\
\text { lndividuen }\end{array}$ \\
\hline & \multicolumn{2}{|c|}{ A.D. +1} & \multicolumn{2}{|c|}{ A D. } & \\
\hline $\begin{array}{c}\text { Année } \\
\text { Jahr }\end{array}$ & $\mathbf{S}^{3}$ & $\mathrm{~F}^{4}$ & $\mathrm{~S}$ & F & \\
\hline $\begin{array}{l}1972 \\
1973 \\
1974\end{array}$ & $\begin{array}{l}5,3 \\
1,9 \\
3,2\end{array}$ & $\begin{array}{r}12,0 \\
4,0 \\
10,4\end{array}$ & $\begin{array}{l}42,9 \\
46,9 \\
40,8\end{array}$ & $\begin{array}{l}39,8 \\
47,2 \\
45,6\end{array}$ & $\begin{array}{r}1029 \\
478 \\
2637\end{array}$ \\
\hline
\end{tabular}

1. Abeilles domestiques visitant "positivement" les fleurs.

2. Abeilles domestiques visitant «négativement 》 les fleurs.

3. et 4. ef. tableau 1.

1. Apis mellifica mit " positivem "Blütenbesuch.

2. Apis mellifica mit " negativem" Blitenbesuch. 3 u. 4 s. Tab 1.

\section{L'alternance de butinage d'une lignée à l'autre}

Quand on suit un individu en train de butiner on voit qu'il visite alternativement des séquences de fleurs mâle-fertile et de fleurs mâle-stérile. Ces séquences peuvent être plus ou moins longues et régulières et on peut envisager de caractériser chaque espèce par les séquences moyennes sur plantes mâlestérile et sur plantes mâle-fertile. D'après le tableau 11 on peut dire que les bourdons ont tous un comportement d'alternance à peu près identique, visitant 
consécutivement en moyenne une vingtaine de fleurs mâle-stérile et une vingtaine de fleurs mâle-fertile. Eucera tuberculata est beaucoup moins fidèle à une lignée puisque l'alternance moyenne est de 6 fleurs mâle-stérile - 8 fleurs mâle-fertile. Quant à l'abeille domestique elle reste 3 fois plus de temps sur la lignée “ mâle » (18 fleurs) que sur la lignée “ femelle » (5 fleurs). Les séquences les plus longues peuvent atteindre 50 ou 100 fleurs mais elles sont peu fréquentes.

TABL. 11. - Fréquence de changement de lignée chez quelques espèces pollinisatrices de la féverole.

Tав. 11. - Häufigkeit im Wechsel der Stämme bei einigen Arten, die die Pferdebohne bestäuben.

\begin{tabular}{|c|c|c|c|c|}
\hline \multirow{3}{*}{$\begin{array}{c}\text { Espèce } \\
\text { Art }\end{array}$} & \multirow{3}{*}{$\begin{array}{l}\text { Nombre } \\
\text { d'individus } \\
\text { observés } \\
\text { Zahl der beob- } \\
\text { achteten Tiere }\end{array}$} & \multicolumn{3}{|c|}{$\begin{array}{c}\text { Nombre de fleurs visitées positivement } \\
\text { Zahl d. positiv. besuchten Blüten }\end{array}$} \\
\hline & & \multirow{2}{*}{$\begin{array}{c}\text { Séquences } \\
\text { Sequenz } \\
\\
\text { Min-Max }\end{array}$} & \multicolumn{2}{|c|}{$\begin{array}{c}\text { séquence moyenne sur les plantes } \\
\text { Mittlere Sequenz } \\
\text { auf d. Pflanzen }\end{array}$} \\
\hline & & & $\begin{array}{l}\text { mâle-stérile } \\
\text { männl.-sterile }\end{array}$ & $\begin{array}{l}\text { mâle-fertile } \\
\text { männl.-fertile }\end{array}$ \\
\hline 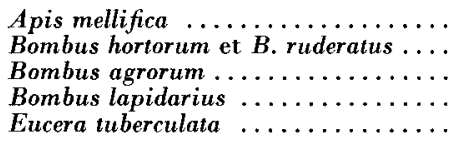 & $\begin{array}{l}14 \\
48 \\
16 \\
22 \\
12\end{array}$ & $\begin{array}{l}1-\quad 55 \\
1-104 \\
2-\quad 88 \\
1-\quad 92 \\
1-\quad 37\end{array}$ & $\begin{array}{r}5 \\
22 \\
16 \\
20 \\
6\end{array}$ & $\begin{array}{r}18 \\
26 \\
19 \\
18 \\
8\end{array}$ \\
\hline
\end{tabular}

1. Séquence $=$ nombre de fleurs de même type (mâle-stérile ou mâle-fertile) visitées consécutivement avant de s'envoler vers l'autre type.

1. Aufeinanderfolge = Anzahl der Blüten gleichen Typs (männlich-steril oder männlich-fertil), die nacheinander besucht werden vor dem Übergang zum anderen Typ

Un changement de comportement de visite est parfois lié au passage d'une lignée à l'autre, par exemple quelques Terrestribombus et abeilles domestiques visitant de façon “ positive » des fleurs mâle-fertile changent de bande et de lignée et visitent de façon ( négative » les fleurs mâle-stérile. Ce changement de comportement peut aussi être observé au cours du butinage d'une série de fleurs de même type (mâle-fertile ou mâle-stérile) chez l'abeille domestique et B. lapidarius.

\section{L'influence du semis en ligne sur le butinage}

Lorsque la féverole commence à fleurir les plantes sont encore basses et la végétation n'étant pas très dense, on distingue parfaitement les lignes de la culture. A ce stade de développement on peut observer que certains individus 
butinent de fleur en fleur en suivant la même ligne. Le nombre de tels insectes est extrêmement réduit en début de floraison et nul lorsque les plantes ont atteint leur hauteur et développement maximum. En effet à ce moment là on ne distingue plus les lignes de la culture.

\section{C. - L'efficacité pollinisatrice des espèces}

L'étude du comportement de butinage exposée dans le paragraphe $B$ permet de porter un jugement d'ensemble sur la valeur pollinisatrice des différentes espèces. Afin de donner une évaluation chiffrée de l'efficacité de chaque espèce on calcule le nombre de fleurs que peut visiter ( positivement ) un individu en 1 minute. L'insecte qui visite positivement une fleur en 1 minute est “ l'insecte unité » ou “ unité de pollinisation » (U.P.). Le tableau 12 donne les coefficients d'efficacité pollinisatrice exprimés en U.P. attribués aux espèces d'apoïdes.

TABL. 12. - Efficacitê pollinisatrice individuelle des apoïdes de la féverole à Lusignan.

TAB. 12. - Individuelle Bestäubungs-Wirksamkeit der Apiden auf Vicia faba equina in Lusignan.

\begin{tabular}{|c|c|}
\hline $\begin{array}{c}\text { Espèce } \\
\text { Art }\end{array}$ & $\begin{array}{c}\text { Coefficient d'efficacité }{ }^{1} \\
\text { pollinisatrice (U.P.) } \\
\text { Kofffzient der Bestäubungs- } \\
\text { Wirksamkeit (U.P.) }\end{array}$ \\
\hline $\begin{array}{l}\text { Apis mellifica } \ldots \ldots \ldots \ldots \\
\text { Bombus terrestris } \boldsymbol{B} \text { lucorum } \\
\text { Bombus hortorum } \text { B ruderatus. } \\
\text { Bombus lapidarius } \ldots \ldots \ldots \ldots \\
\text { Bombus agrorum. } \ldots \ldots \ldots \ldots \\
\text { Bombus pratorum } \ldots \ldots \ldots \ldots \\
\text { Bombus sylvarum } \ldots \ldots \ldots \ldots \\
\text { Eucera tuberculata } \ldots \ldots \ldots \\
\text { Anthophora acervorum } \ldots \ldots \ldots\end{array}$ & $\begin{array}{r}0,6 \\
0,8 \\
12,8 \\
7,7 \\
5,9 \\
3,4 \\
8,7 \\
6,6 \\
5,8\end{array}$ \\
\hline
\end{tabular}

1. Un individu visitant positivement une fleur par minute $=1$ unité de pollinisation (U.P.). Chaque coefficient indique le nombre d'U.P. représentées par un individu de chaque espèce.

1. Ein in einer Minute eine Blüte positiv besuchendes Individuum $=1$ Bestäubungseinheit (U.P.). Jeder Koeffizient gibt die Zahl der Bestäubungseinheiten an, die von einem Tier erbracht wird.

L'abeille domestique et les Terrestribombus se distinguent par leur faible efficacité tandis que les Hortobombus sont particulièrement actifs (15 à 20 fois plus que les précédents). Toutes les autres espèces sont intermédiaires. Il faut 
bien noter que le coefficient de l'abeille domestique doit être réajusté selon l'année, le lieu et la lignée : il peut varier de 0,1 à 1 .

L'estimation de l'efficacité individuelle permet de pondérer les densités de population évaluées après les dénombrements d'insectes et de calculer le nombre d'unités de pollinisation que fournit chacune des espèces. On voit ainsi que l'abeille domestique représentant 70 à $90 \%$ de la faune des apoïdes sur les plantes mâle-stérile, n'effectue que 11 à $42 \%$ du total des visites ( positives ») sur la lignée “ femelle » (tableau 13). Si l'on considère uniquement la faune sauvage on voit sur le tableau 14 qu'en moyenne sur 4 ans à Lusignan, plus de $70 \%$ de la pollinisation de la féverole sont assurés par : les Hortobombus, B. lapidarius, Eucera tuberculata. Les Hortobombus et B. lapidarius sont les espèces sauvages les plus importantes du point de vue économique et les plus constantes (tableau 15 et fig. 13).

TABL. 13. - Importance numérique et efficacité pollinisatrice relative de l'abeille domestique suivant l'année et le type de la lignêe de féverole visité à Lusignan.

TAB. 13. - Zahlenmässige Bedeutung und relative Bestäubungs-Wirksamkeit von Apis mellifica nach Jahr und Stamm der besuchten Pferdebohne in Lusignan.

\begin{tabular}{|c|c|c|c|c|c|c|}
\hline \multirow{3}{*}{$\begin{array}{l}\text { Année } \\
\text { Jahr }\end{array}$} & \multicolumn{2}{|c|}{1972} & \multicolumn{2}{|c|}{1973} & \multicolumn{2}{|c|}{1974} \\
\hline & \multicolumn{6}{|c|}{$\begin{array}{c}\text { Densité moyenne de la faune par jour et par hectare. } \\
\text { Mittlere Dichte der Fauna je Tag und ha }\end{array}$} \\
\hline & $\mathrm{N}^{1}$ & $\%$ A.D. ${ }^{2}$ & $\mathbf{N}$ & $\%$ A.D. & $\mathbf{N}$ & $\%$ A.D. \\
\hline \multirow[t]{3}{*}{$\begin{array}{l}\text { F }^{3} \\
\mathrm{~S}^{4}\end{array}$} & $\begin{array}{ll}1 & 142 \\
1 & 050\end{array}$ & $\begin{array}{l}70,6 \\
71,1\end{array}$ & $\begin{array}{l}745 \\
757\end{array}$ & $\begin{array}{l}82,1 \\
76,9\end{array}$ & $\begin{array}{l}3769 \\
3059\end{array}$ & $\begin{array}{l}93,1 \\
90,4\end{array}$ \\
\hline & \multicolumn{6}{|c|}{$\begin{array}{c}\text { Travail de pollinisation de la faune } \\
\text { Bestäubungsarbeit der Fauna }\end{array}$} \\
\hline & U.P. ${ }^{5}$ & $\begin{array}{l}\% \text { A.D. } \\
\text { (U.P.) }\end{array}$ & U.P. & $\begin{array}{c}\% \text { A.D. } \\
\text { (U.P.) }\end{array}$ & U.P. & $\begin{array}{l}\% \text { A.D. } \\
\text { (U.P.) }\end{array}$ \\
\hline $\begin{array}{l}\mathbf{F} \\
\mathbf{S}\end{array}$ & $\begin{array}{l}2575 \\
1659\end{array}$ & $\begin{array}{l}34,4 \\
23,9\end{array}$ & $\begin{array}{l}773 \\
974\end{array}$ & $\begin{array}{l}29,2 \\
11,3\end{array}$ & $\begin{array}{ll}4 & 158 \\
2 & 304\end{array}$ & $\begin{array}{l}75,1 \\
42,0\end{array}$ \\
\hline
\end{tabular}

1. Nombre total d'apoïdes.

2. Pourcentage d'abeilles domestiques du nombre total des apoïdes.

3. et 4. Cf. tableau 1 .

5. Transformation de la donnée $N$ en unités de pollinisation (U.P.), c'est-à-dire en nombre de fleurs déclenchées en moyenne par hectare et par minute.

6. Pourcentage de déclenchement en U.P. dû̀ à l'abeille domestique.

1. Gesamtzahl der Apiden.

2. Prozentualer Anteil von Apis mellifica an der Gesamtzahl der Apiden.

3. u. 4. siehe Tabelle 1 .

5. Umwandlung des Wertes $\mathrm{N}$ in Bestäubungs-Einheiten (U.P.), d.h. in die Zahl der im Mittel je ha und Minute aufgeklinkten Blüten. 
TABL. 14. - Importance numérique et efficacité pollinisatrice relatives des populations d'apoïdes sauvages à Lusignan. $1971-72-73-74$.

TAB. 14. - Zahlenmässige Bedeutung und relative Bestäubungswirksamkeit der wilden Apiden-Populationen in Lusignan 197/-72-73-74.

\begin{tabular}{|c|c|c|}
\hline $\begin{array}{c}\text { Espèce } \\
\text { Art }\end{array}$ & $N^{1}$ & U.P. 2 \\
\hline 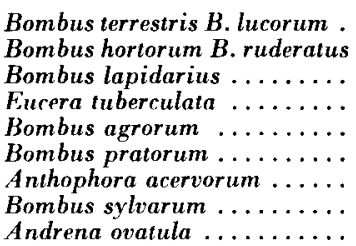 & $\begin{array}{c}36,6(40,0) \\
17,0(16,8) \\
12,8(9,9) \\
11,9(19,4) \\
8,7(2,6) \\
5,9(2,0) \\
3,8(3,4) \\
3,1(2,8) \\
\end{array}$ & $\begin{array}{r}5,4 \\
40,0 \\
18,1 \\
14,5 \\
9,4 \\
3,7 \\
4,0 \\
5,0 \\
-\end{array}$ \\
\hline
\end{tabular}

1. Pourcentage du nombre total d'apoïdes sauvages. (Domnées entre parenthèses) Pourcentage moyen du nombre total des apoïles sauvages recensés en 1973 dans 19 champs de féverole répartis dans toute la France.

2. Pourcentage du nombre total d'unités de pollinisation.

1. Prozentsatz der Gesamtzahl wilder Apiden. (Daten in Klammern) Mittlerer Prozentsatz der Gesamtzahl wilder Apiden, die auf 19 über ganz Frankreich verteilten Vicia faba-Feldern festgestellt wurden; 1973.

2. Prozentsatz der Gesamtzahl von Bestäubungs-Einheiten.

TABL. 15. -- Importance pollinisatrice relative des espèces d'apoïdes sauvages à Lusignan en fonction de l'année.

ТАВ. 15. -... Relative Bedeutung der wilden Bienenarten für die Bestäubung abhängig vom .Jahr.

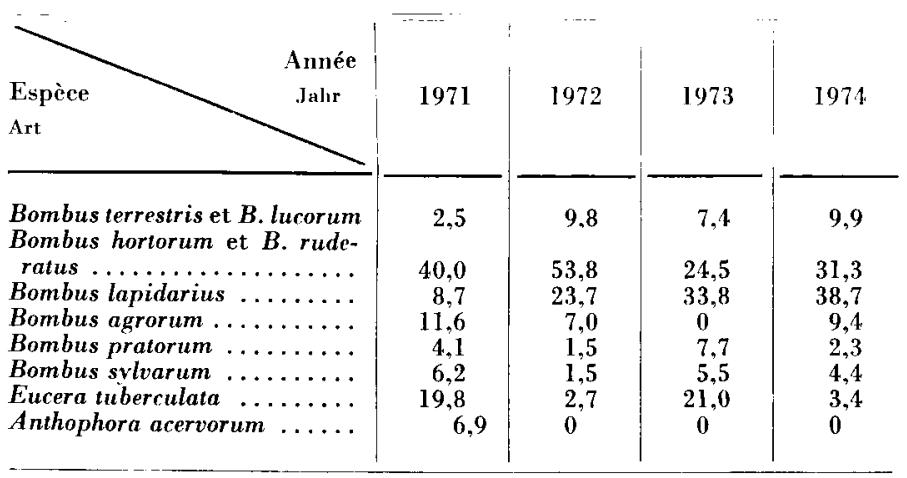

Les nombres représentent les pourcentages du total des fleurs déclenchées chaque année par la faune sauvage.

Die Zahlen geben die Prozente der jedes Jahr durch die wilden Bienen aufge. klinkten Bliiten an. 


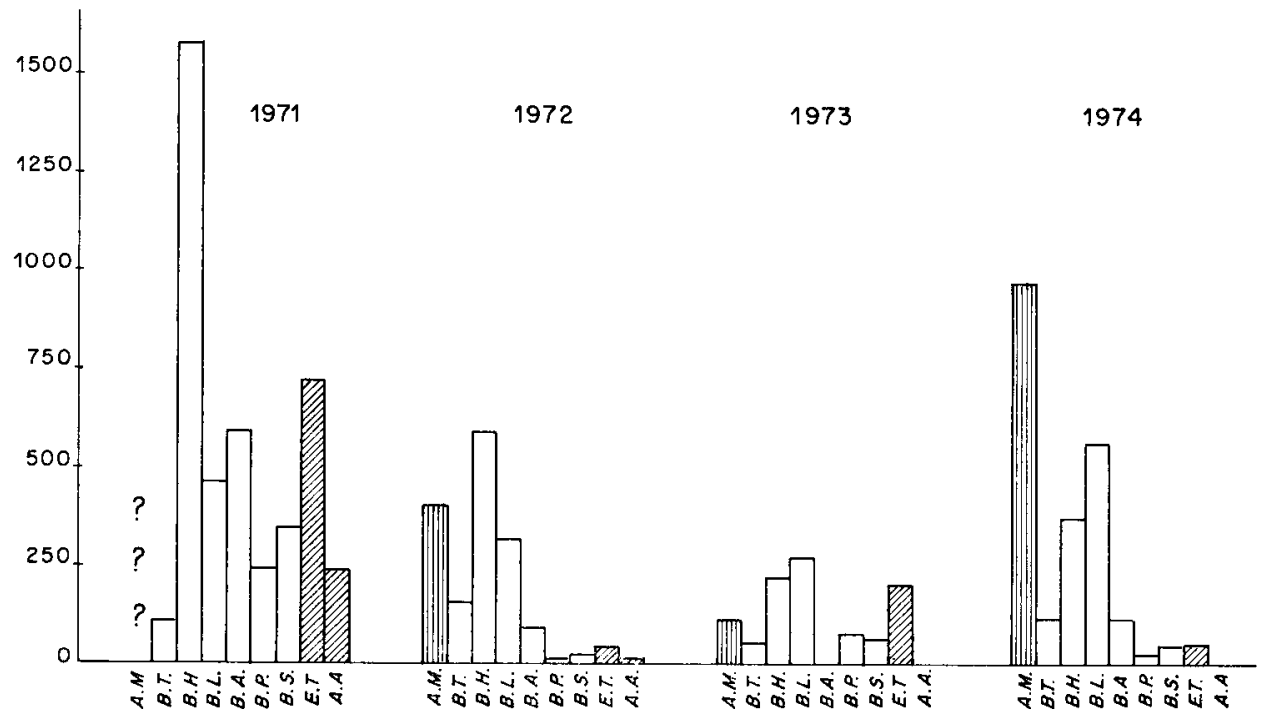

Fic. 13. - Pollinisation des plantes mâle-stérile de féverole par les Apoidea, à Lusignan. Verticalement : Nombre d' « unités de pollinisation » par hectare et par jour.

Авв. 13. - Bestäubung der männlich-sterilen Pfanzen von Vicia faba durch die Apiden in Lusignan Ordinate $=$ Anzahl « Bestäubung-Einheiten » je Tag und ha

Apoïdea solitaires solitäre Apoidea

B.T. Terrestribombus

B.H. Hortobombus

B.L. Bombus lapidarius

B.A. Bombus agrorum

B.P. Bombus pratorum

B.S. Bombus sylvarum

E.T. Eucera tuberculata

A.A. Anthophora acervorum

Bombus

WDIII Apis mellifica 


\section{D. - Étude particulière de l'abeille domestique La récolte des pollens}

Les figures 14, 15 et 16 montrent que le pollen de féverole ne représente en moyenne que 15 à $30 \%$ de la récolte totale de pollen. Certaines plantes détournent une proportion très importante d'abeilles; suivant l'année il s'agit surtout de Crucifères (Brassica napus) et Rosacées (Pirus, Prunus). De façon moins marquée d'autres plantes peuvent aussi intéresser les butineuses de pollen : Vicia sp., Papaver Rheas, Ranunculus sp., Quercus sp.

Ruche $n^{\circ} \mathrm{y}$

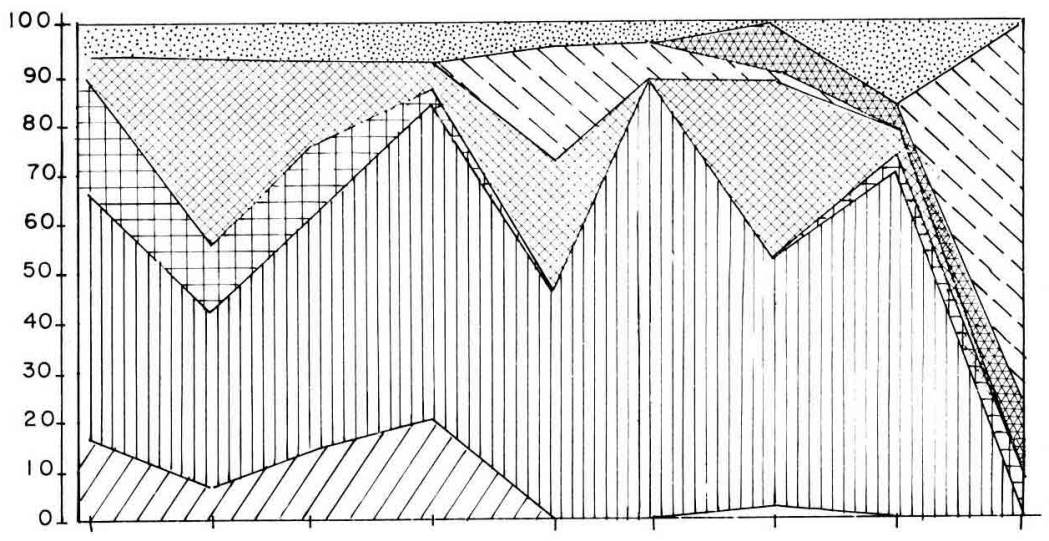

A vicio fobo

पाIIII] Cruciferae

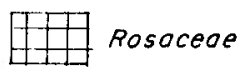

E\ר popilionaceoe

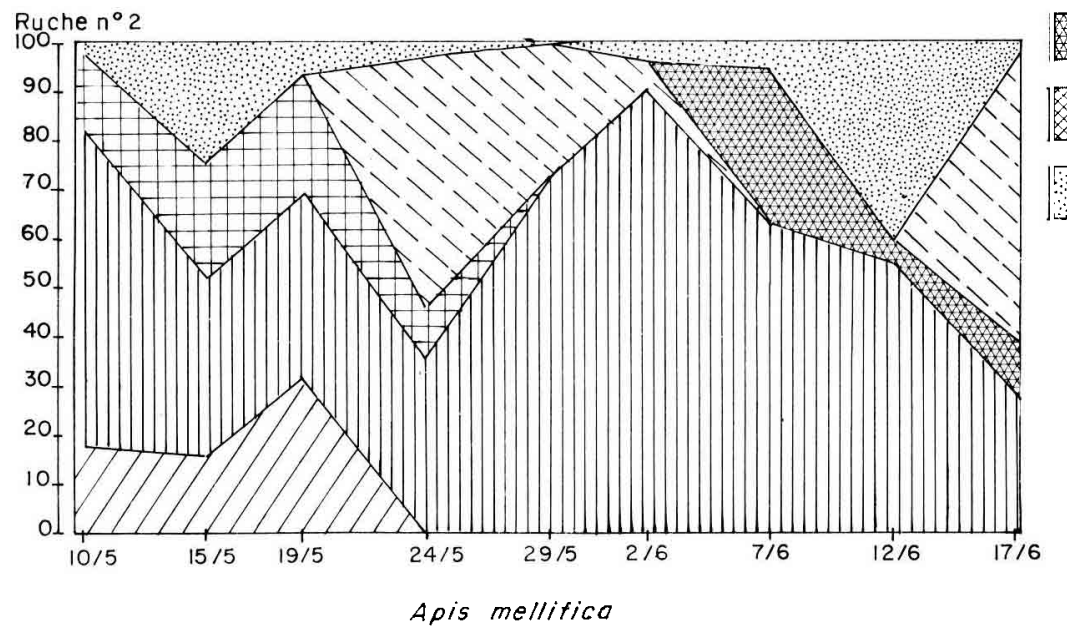

Papaveraceae

Renonculaceae

Divers

FIG. 14. - Récolte des pollens par l'Abeille domestique. Lusignan 1972.

Verticalement $=$ pourcentage du nombre total de pelotes dans un échantillon de 400 pelotes de pollen.

Horizontalement $=$ Date du prélèvement

Aвв. 14. - Pollenernte von Apis mellifica. Lusignan 1972

Ordinate $=\%$ der Gesamtzahl von Pollenhöschen in einer Probe von 400 Pollenhöschen Abszisse $=$ Datum der Ermittlung 


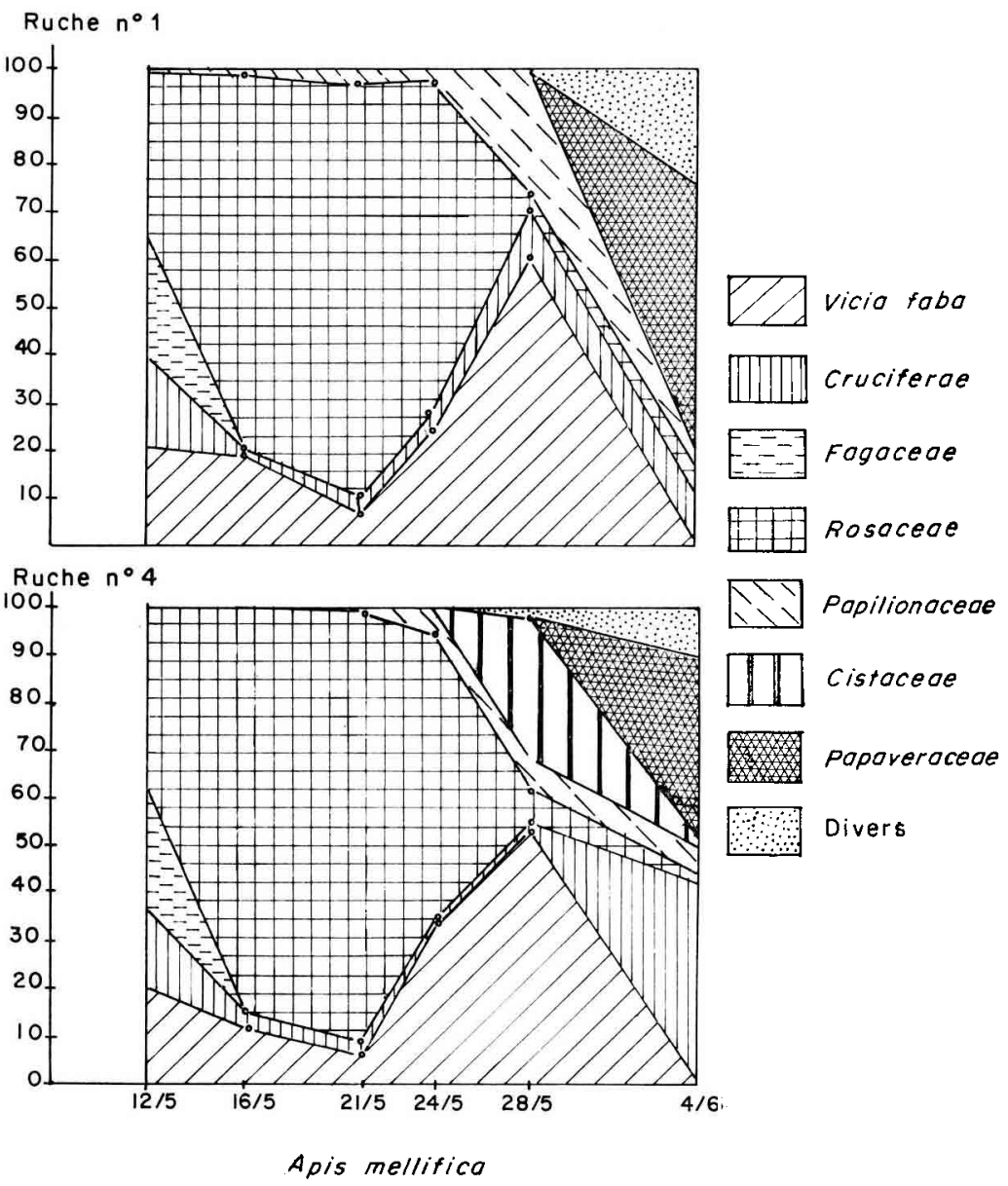

Fig. 15. - Récolte des pollens par l'Abeille domestique. Lusignan 1973. Même légende que Fig. 14.

AвB. 15. - Pollenernte von Apis mellifica, Lusignan 1973

Legende wie in Abb. 14 

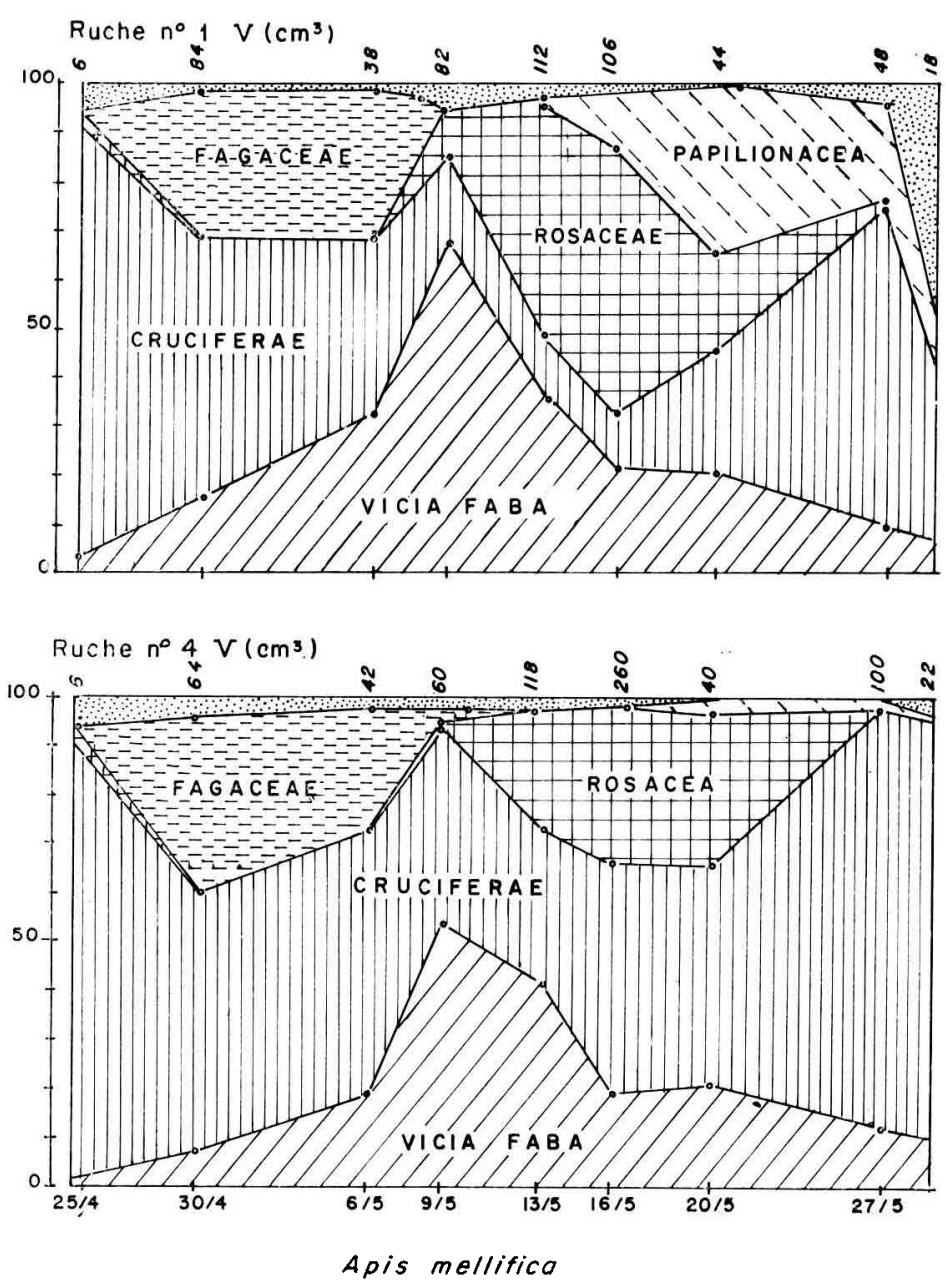

Fig. 16. - Récolte des pollens par l'Abeille domestique. Lusignan 1974.

Même légende que Fig. 14 .

$\mathrm{V}\left(\mathrm{cm}^{3}\right)=$ Volume total des pelotes tombées dans la trappe à pollen exprimé en centimètres cube.

Авв. 16. - Pollenernte von Apis mellifica, Lusignan 1974

Legende wie in Abb. 14

$\mathrm{V}\left(\mathrm{cm}^{3}\right)=$ Gesamtvolumen der Pollenhöschen in der Pollenfalle in $\mathbf{c m}^{3}$

Lorsque l'on fait 2 relevés de trappe à pollen chaque jour, l'un à 12 heures, l'autre en fin de soirée on constate que la proportion de pelotes de Vicia faba est très faible le matin et beaucoup plus élevée l'après-midi (tableau 16). 
TABL. 16. - Rêcolte du pollen de Vicia faba par l'abeille domestique en fonction du moment de la journée - Lusignan 1972

TAB. 16. - Pollenernte von Apis mellifica aus Vicia faba in Abhängigkeit von der Tageszeit; Lusignan 1972

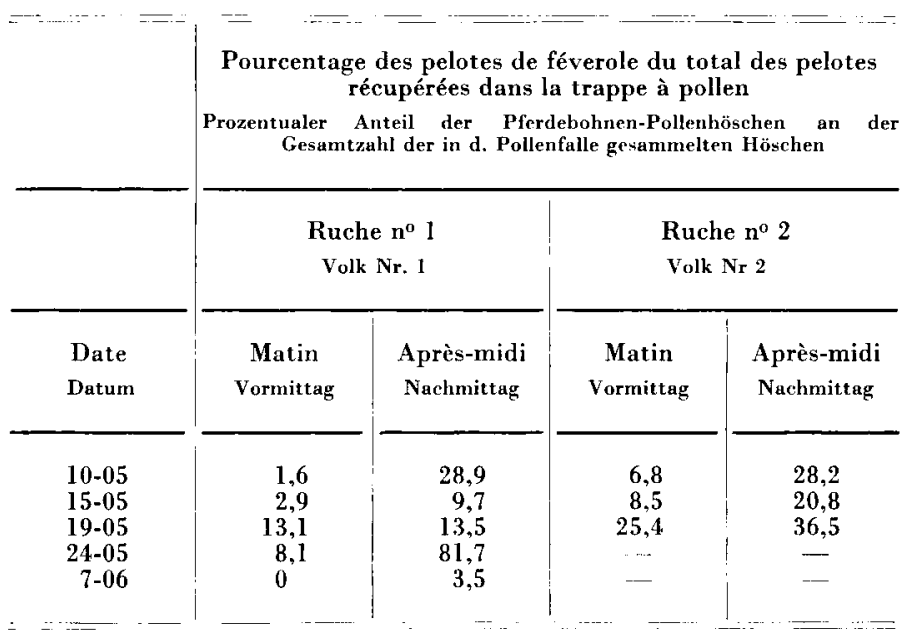

Le fait de nourrir les colonies avec du sirop sucré pendant la floraison n'a pas incité les 6 ruches testées en 1973 et 1974 à récolter plus de pollen de féverole.

\section{E. - Pollinisation des plantes mâle-stérile Dans divers dispositifs culturaux}

Je fais part ici de résultats obtenus en 1974 par A. Delaude et non publiés. Trois types de dispositifs de bandes alternées " mâle 》 et “ femelle 》 sont testés dans les mêmes conditions. Ils sont installés côte à côte dans une dizaine de champs dont 7 renferment la même lignée “ $48 \mathrm{~B}$ ” qui a un taux de restauration de fertilité ( mâle » variant de 11 à $42 \%$.

La nouaison exprimée en nombre de gousses par tige varie de 5,2 à 15,1 (moyenne : 7,5) dans la ligne médiane du dispositif $\mathrm{n}^{\circ} 3$ (bande " femelle 》 d'une largeur de 7,5 m). Dans le dispositif $n^{\circ} 1$ (bande ( femelle » d'une largeur de $2,5 \mathrm{~m}$ ) la nouaison de la ligne médiane varie de 5,3 à 11,1 (moyenne : 7,5). L'analyse statistique globale ne fait pas ressortir d'influence du dispositif sur la nouaison des lignes médianes. Un accroissement de la largeur des bandes “ mâle stérile » du parent femelle, dans les limites $2,5 \mathrm{~m}-7,5 \mathrm{~m}$, ne nuit pas à la pollinisation de la partie médiane de ces bandes. 
Le rendement grainier du parent femelle exprimé en pourcentage du rendement grainier du parent mâle varie de 30 à $142 \%$ (moyenne : $63 \%$ ) et on ne décèle aucune influence de la largeur des bandes a femelle ».

Dans les conditions expérimentales de 1974 aucune diminution de la nouaison et du rendement grainier n'est imputable à un déficit de pollinisation dans la partie médiane des bandes ( femelle » de grande largeur (7,5 m). Cependant il est possible que la même expérience conduite avec une lignée " femelle » plus pure ayant un taux de restauration de fertilité (c mâle » inférieur à $10 \%$ \% n'aboutisse pas au même résultat. D'autres essais entrepris avec un matériel végétal nouveau sont donc à prévoir; ils nous informeront d'une façon plus précise sur l'influence du dispositif de culture sur la fructification et le rendement.

\section{DISCUSSION}

\section{1. - Sur les spectres de faune pollinisatrice de Vicia faba}

L'abeille domestique est pour la plupart des auteurs l'insecte pollinisateur le plus abondant sur la féverole et la fêve, les bourdons ne représentant que 10 à $20 \%$ de la faune. Ceci correspond aux estimations données dans cet article. Les abeilles solitaires ne font pas souvent l'objet de remarques et seuls WAFA et Mohamed (1970) les mentionnent dans leur inventaire des apoïdes de la fêve en Égypte, ainsi que Pritsch (1971). Les auteurs égyptiens citent 6 espèces d'Anthophoridae appartenant aux genres : Tetralonia, Anthophora et Eucera; cette observation confirme l'attirance de cette famille d'abeilles solitaires terricoles pour Vicia faba.

Parmi les bourdons, les Terrestribombus sont toujours mentionnés et souvent comme les plus abondants (80\% de la faune sauvage au Danemark d'après Poulsen - 1973). Par contre en Grande-Bretagne Bond et Hawkins (1967) trouvent que les Hortobombus sont les bourdons les plus nombreux. En France le groupe d'abeilles sauvages le plus important est celui des Terrestribombus ( $40 \%$ de la faune sauvage) alors que celui des Hortobombus ne représente que $20 \%$ environ de cette faune. Ces proportions sont assez constantes quelque soit le lieu et l'année.

\section{2. - Sur le comportement de butinage des espèces}

Le percement de la base des fleurs de fêve et de féverole par les Terrestribombus et l'utilisation des orifices artificiels ainsi créés par d'autres espèces est un phénomène constamment observé et souvent commenté par les auteurs s'intéressant à la pollinisation de Vicia faba. Scriven et al (1961) observent des ouvrières d'abeille domestique perçant des trous dans des corolles de $V$. faba 
mais il s'agit là d'un comportement atypique survenant sous cage. BriaN (1954 et 1957) relate le percement de fleurs d'Erica, Althea, Lathyrus, Lotus par Bombus lucorum et estime qu'il s'agit d'une réponse instinctive adaptée seulement à certaines espèces végétales. Cet auteur pense que l'utilisation des trous par les autres espèces d'apoïdes est liée à la longueur de leur langue. En effet le butinage “ négatif ) chez la féverole est observé chez les espèces possédant une langue d'une longueur inférieure à $12 \mathrm{~mm}$. Cette longueur limite est celle des langues des Reines de Bombus lapidarius et $B$. agrorum qui sont assez peu fréquemment « négatives ». Lorsque la longueur de langue est réduite à $10 \mathrm{~mm}$ (cas de $B$. pratorum) et surtout à $6-7 \mathrm{~mm}$ (cas de l'abeille domestique) la proportion des individus “ négatifs » augmente considérablement. Les Hortobombus à langue longue (16 mm chez les Reines) ont un comportement stéréotypé et ils n'utilisent jamais les trous. Les Terrestribombus et les Hortobombus sont donc des types extrêmes quand on considère la variabilité du comportement de butinage des fleurs de féverole. Poulsen (1971) estime que l'utilisation des trous par un nombre croissant d'ouvrières d'abeille domestique au cours de la floraison est le signe d'un apprentissage. La similitude des résultats obtenus en France, et leur répétition renforcent cette opinion qui était déjà partagée par Brandenburg (1961). Cet auteur recommande en conclusion un remplacement fréquent des ruches afin d'en obtenir le meilleur rendement dans la pollinisation de Vicia faba. L'apprentissage du butinage négatif est certainement une explication du changement de comportement de Bombus lapidarius dans la deuxième moitié de la floraison.

Les visites stipulaires effectuées par l'abeille domestique paraissent beaucoup plus fréquentes dans les conditions anglaises et danoises que dans les conditions françaises, surtout en fin de floraison puisqu'à ce moment 75 à $80 \%$ des ouvrières peuvent être détournées des fleurs (Free 1962 et Poulsen 1973).

La grande différence de fréquentation de la féverole par l'abeille domestique le matin et l'après-midi est remarquée par plusieurs auteurs. FreE (1962) et Poulsen (1973) situent entre 14 et 17 heures la période de grande affluence des abeilles butineuses de nectar et de pollen. Poulsen (1971) dénombre à peu près 3 fois plus d'ouvrières l'après-midi que le matin et trouve que les résultats des comptages effectués sur colza (Brassica napus) sont l'inverse des précédents. L'explication du phénomène est due à Percival (1955) qui note que chez Vicia faba le pollen est libéré surtout entre 12 et 15 heures (90\% des étamines) et que les $3 / 4$ des boutons floraux s'ouvrent entre 12 et 14 heures.

Bond et Hawkins (1967) seuls auteurs à observer le butinage des insectes dans une culture mixte en production d'hybride, constatent que sur les plantes mâle-fertile environ $22 \%$ des ouvrières d'abeille domestique sont “ positives » contre $2,5 \%$ seulement sur les plantes mâle-stérile. La désaffection des butineuses « positives » à l'égard des plantes mâle-stérile est ainsi démontrée d'une 
manière encore plus évidente qu'à Lusignan. Les auteurs britanniques n'ont jamais pu observer une ouvrière transporter du pollen des plantes “ mâle » vers les plantes “ femelle ». A la suite de ces chercheurs j’émets beaucoup de réserves sur l'efficacité pollinisatrice de l'abeille domestique dans le cas des cultures mixtes productrices de semence hybride de féverole. Certaines données de cet article indiquent qu'avec de fortes populations d'abeilles domestiques (blus de 6000 individus par hectare) un nombre considérable de croisements est dû à cette espèce $(40 \%)$. Or cette estimation de la part prise par l'abeille domestique dans la pollinisation des plantes mâle-stérile est probablement surestimée. En effet Berthelem (communication personnelle) pense que les abeilles domestiques butinent “ positivement ” de préférence, les fleurs déjà visitées par d'autres espèces, car les fleurs “ vierges » de féverole offrent une résistance relativement grande lors de la première ouverture. Ce phénomène, ainsi que la fréquentation massive des plantes l'après-midi viennent réduire la probabilité de c réussite » d'une visite positive d'abeille domestique.

Afin de pallier la faible efficacité des abeilles domestiques on peut accroître le nombre des individus. Cependant on n'est jamais sûr du résultat d'un apport de ruches car les plantes compétitrices : Trèfles, Crucifères (Poulsen 1971 - Ste uCKardT 1965), Rosacées (observations de Lusignan) drainent une partie importante des butineuses : $80 \%$ de la population de Lusignan, 15 à $90 \%$ selon les conditions expérimentales de divers auteurs (FrEe, 1970). Quant à l'accroissement artificiel de la récolte de pollen de Vicia faba par nourrissage au sucre des ruches $(300 \%$ d'après FrEe - 1965) il n'a pu être obtenu à Lusignan de façon constante et bien significative.

La variabilité des paramètres de l'efficacité pollinisatrice de l'ouvrière et de la ruche d'abeille domestique ne permet pas de porter un jugement décisif sur l'intérêt d'apporter des colonies dans les champs de production de semence hybride de féverole. Dans les conditions françaises actuelles les principaux responsables de la production de semence hybride de féverole sont les Bourdons à langue longue (Hortobombus, B. lapidarius, B. agrorum) et les abeilles solitaires du genre Eucera. Ces insectes, qui effectuent les croisements souhaités, sont porteurs d'une masse de pollen du parent mâle, qui se réduit au cours des visites sur le parent femelle. Les 10 premières visites sont probablement les seules qui ont quelque chance d'être fécondantes. Le “ rendement pollinisateur » des populations d'apoïdes est donc inférieur dans le dispositif de cultures à bandes “ mâle » et “ femelle » alternées, à celui des populations qui visitent la féverole classique possédant des fleurs hermaphrodites c'est pourquoi on doit veiller à disposer d'une densité suffisante d'espèces bonnes pollinisatrices.

\section{CONCLUSIONS}

Dans l'état actuel de pureté des lignées mâle-stérile de féverole l'alternance $2,5 \mathrm{~m}$ de plantes “ mâle » et $7,5 \mathrm{~m}$ de plantes “ femelle " permet aux bandes mâle-stérile d'être pollinisées de façon homogène sur toute la largeur. 
Bien que l'abeille domestique représente en moyenne $80 \%$ des insectes butineurs, ce sont des espèces d'apoïdes sauvages relativement abondants et efficaces : Hortobombus, B. lapidarius, Eucera tuberculata qui assurent la majeure partie de la production de graines hybrides. Les populations d'abeille domestique ont une efficacité très réduite en moyenne. Cependant les résultats diffèrent sensiblement d'une année à l'autre pour des raisons multiples et parfois difficiles à saisir. Généralement l'efficacité pollinisatrice de l'abeille domestique est liée à la présence des Terrestribombus et à l'utilisation de leurs trous dans les fleurs, ainsi qu'à l'attirance des butineuses de pollen pour les plantes mâle-fertile. Par ailleurs les effets d'apports de ruches sont très atténués à cause de la présence et la concurrence de plantes mellifères ou pollinifères : Crucifères, Rosacées, Cupulifères... Il semble donc que dans les conditions actuelles on ne puisse guère compter sur cette espèce pour améliorer la production de semence hybride à cause de son efficacité à la fois faible et variable. Seule l'absence de bourdons perceurs de corolles permettrait l'utilisation de l'abeille domestique puisque les butineuses de nectar deviendraient " positives » et participeraient à la pollinisation sans marquer de préférence pour la lignée mâle comme c'est le cas pour les butineuses de pollen.

Dans nos conditions écologiques il paraît conseillé d'installer des champs de production d'hybride de dimension modeste pour ne pas c diluer " la faune sauvage, dans des lieux favorables à la prolifération des bourdons c'est-à-dire les zones boisées, riches en friches, prairies permanentes et cultures mellifères à floraisons échelonnées.

Reçu pour publication en octobre 1975.

Eingegangen im Oktober 1975.

\section{REMERCIEMENTS}

Je remercie Monsieur A. Defaude et la Fédération Nationale des Multiplicateurs de Semences pour leur collaboration tout au long de ce travail ainsi que les Établissements Multiplicateurs de Semences qui ont participé à une enquête faunistique régionale.

Mes remerciements vont aussi à Messieurs P. Berthelem et J. Picard pour l'intérêt qu'ils ont manifesté lors de cette étude.

\section{ZUSAMMENF ASSUNG}

Die Nutzbarmachung neuerer Untersuchung enüber die “ männlich-sterile » Pferdebohne, die in Frankreich und England durchgeführt wurden, sollte die Erzeugung von KreuzungsVarietäten ermöglichen, die ertragreicher sind als die derzeitig gezüchteten Varietäten. Die vorliegende Arbeit ist ein Beitrag zur Untersuchung der Möglichkeiten, Hybrid-Samen nnter den Bedingungen der landwirtschaftlichen Praxis zu erzeugen, d.h. auf Feldern, die streifenr weise abwechselnd mit " weiblichen " (männlich-sterilen) und " männlichen " (männlichfertilen) Pflanzen angelegt sind. Hauptsächlich wurde das Sammelverhalten der verschiedenen 
Bienenarten untersucht, ihre Zahl berechnet und ihre Wirksamkeit bei der Bestäubung ausgewertet.

\section{Material und Methode}

Die Beobachtungen wurden vier Jahre lang (1971-1974) hauptsächlich an den Mischkulturen der Winter-Pferdebohne in Lusignan, Departement Vienne durchgeführt (Abb. 1) Die Felder sind mit einem männlichen und einem nicht ganz rein-weiblichen Stamm bestellt. Von etzterem waren $7-20 \%$ der angeblich männlich-sterilen Pflanzen tatsächlich männlich-fertile (Tab. 1). 18 andere Felder, die durchschnittlich 3 ha gross und auf sieben französische Departements verteilt waren, bildeten 1973 die Grundlage einiger weiterer Untersuchungen. Diese Felder waren entweder mit P flanzen des herkömmlichen Typus oder eines Mischtypus bestanden. Der Lage entsprechend wurde der " weibliche "Same in Breiten von 2,5 m, $5 \mathrm{~m}$ oder 7,5 m ausgesät, während der “ männliche » Same immer in einer Breite von 2,5 m ausgesät wurde. Die einzelnen Reihen hatten einen Abstand von ungefähr 0,4 m.

Die Insekten wurden entweder zweimal am Tage gezählt, einmal vormittags und einmal nachmittags (1972) oder nur einmal täglich, abwechselnd vor-und nachmittags. Die Fläche, über der gezählt wurde, umfasst etwa $200 \mathrm{~m}^{2} \mathrm{~b}$ lühender Pflanzen $\left(100 \mathrm{~m}^{2}\right.$ männlich-fertile $100 \mathrm{~m}^{2}$ männlich-sterile Pflanzen). Auf dieser Fläche wurden in der Diagonale des Feldes acht $25 \mathrm{~m}$ lange Anteile zu je zwei nebeneinander liegenden Reihen verteilt. Bei jedem Begehen des Feldes wird auf die Art des bestäubenden Insekts, die Kaste, etwa vorhandene Pollenhöschen und die Art, wie der Rüssel eingeführt wird, geachtet. (Natürliche Einführung in die Blumenkrone wird als “ positiver » Besuch gewertet, Einführung durch ein Loch an der Blütenbasis gilt als « negativer » Besuch.

Eine eingehende Untersuchung des Verhaltens wird an wahllos herausgegriffenen Einzeltieren auf dem Feld durchgeführt. Jedes Insekt wird verfolgt, seine Zeit gemessen, die Anzahl der besuchten Blüten, die Art des Besuches (positiv oder negativ) und der Stamm der betreffenden Pflanze festgestellt. Zudem wurden Bienenvölker (Apis mellifica) eingesetzt, deren Kästen mit Pollenfallen ausgestattet waren. Die Völker wurden zum Teil mit $50 \%$ igem Zuckkerwasser gefüttert und teilweise nicht gefüttert, um den Ein fluss des Fütterns auf die Pollen. sammeltätigkeit zu untersuchen. Die Pollenfallen waren an zwei Tagen in der Woche in Betrieb. Die Pollenanalyse wurde an Proben von 300 bis 400 Pollenhöschen durchgeführt.

1974 wurden etwa zehn Versuchsfelder dazu benutzt, den Einfluss der Streifenbreite der weiblichen Pflanzen auf die Bestäubung in den Mittelstreifen zu untersuchen. Wenn die Hülsen reif sind, wird der Fruchtansatz an den Stengeln errechnet und die einzelnen " weiblichen » Reihen je nach Breite gesondert abgeerntet.

\section{Ergebnisse}

\section{Verzeichnis der bestäubenden Fauna}

Apis mellifica ist die am häufigsten vorkommende Art (80\% der Apiden), Hummeln sind dreimal so stark vertreten wie die solitären Bienen. Diese wilden Bienen treten durchschnittlich je Tag und ha in einer Stärke von 500 Individuen auf. Unter den Hummeln sind die Erdhummeln am häufigsten (Bombus lucorum, B. terrestris). Hortobombus sind die zweitwichtigste Gruppe; B. lapidarius, B. sylvarum, B. agrorum, B. pratorum sind seltener. Die am häufigsten vorkommenden solitären Bienen sind Eucera tuberculata und Anthophora acervorum. Vereinzelt treten auch Andrena ovatula und A. flavipes auf (Abb. 3 und 4; Tab. 3).

\section{Das Sammelverhalten}

Die Apiden, die die von Hummeln geschaffenen Löcher benutzen, gehören zu den Arten mit kurzem oder mittellangem Rüssel : Apis mellifica (80-95\% der “ negativen 》 Besucher), 
Bombus pratorum (70 \%) B. lapidarius und B. agrorum (10-15\%). Hortobombus, Hummeln mit langem Rüssel, Eucera und Anthophora benutzen diese künstlich geschaffenen Eingänge niemals und sind hundertprozentige Bestäuber (Abb. 7 bis 12). Die Tiere mit der Tendenz zu c negativem " Verhalten werden immer zahlreicher, je näher das Ende der Blütezeit kommt; es hat den Anschein, als ob diese Art des Sammelns gelernt würde. Die extrafloralen Nektarien werden gegen Ende der Blütezeit von einigen Arbeiterinnen von Apis mellifica besucht sowie von einer solitären Biene : Andrena carantonica, die niemals die Blüten aufsucht.

- Die schnellsten Insekten sind Hortobombus (13 Blïten in der Minute), die langsamsten Apis mellifica, besonders beim Pollensammeln (4 Blüten in der Minute).

- Der Besuch des Feldes ist bei Apis mellifica nachmittags dreimal so intensiv wie am Vormittag; bei den Hummeln dagegen nur 1 1/2 mal so stark (Tab. 8)

- Im Ganzen genommen neigen Apis mellifica, Hortobombus und Eucera dazu, den männlich-fertilen Stamm zu bevorzugen. Man kann feststellen, dass die Pollensammlerinnen von Apis mellifica (“ positive " Arbeiterinnen) dreimal so zahlreich auf den männlich-fertilen Pflanzen wie auf den männlich-sterilen anzutreffen sind. Die Besuche des weiblichen Stammes scheinen “ Irrtümer 》 zu sein. Eine Pollensammlerin besucht tatsächlich nacheinander im Mittel 18 Blüten des männlichen Stammes gegen 5 des weiblichen. Den Hummeln ist allen eine ( symmetrische » Alternativ-Sammelweise zu eigen, etwa 20 Blüten von jedem Stamm. Eucera wechselt die Stämme leichter (6 Blüten auf weiblichen Pflanzen, dann 8 auf männlichen) Siehe dazu Tab. 2.

\section{Die Bedeutung der Arten für die Bestäubung der männlich-fertilen Pflanzen}

Wenn man das Insekt, das 1 Blüte in einer Minute " positiv " besucht, als " InsektenEinheit » oder “ Bestäubungs-Einheit » bezeichnet, kann man jeder Art einen Koeffizienten, ausgedrückt in “ Bestäubungs-Einheiten » zuschreiben (Tab. 12). Apis mellifica und Erdhummeln zeichnen sich durch schwache Wirksamkeit aus, wahrend Hortobombus besonders aktiv sind (15-20 mal mehr als Apis mellifica). Die Aktivität der anderen Arten liegt dazwischen. Durch ein Abwägen der Populationen nach diesen Koeffizienten lässt sich die Bestäubungsleistung der Apiden abschätzen. (Tab. 13, 14, 15, Abb. 13). Wenn man die Zahl der Sammlerinnen von Apis mellifica vergrössern möchte, um ihre geringe Leistung zu verbessern, stellt man fest, dass ein Grossteil der Bienenvölker $(70-85 \%)$ von Nektar liefernden Pflanzen wie Cruciferen, Rosaceen und anderen angelockt werden (Abb. 14, 15, 16). Das Verfüttern von Zuckerwasser hat die Pollenernte aus der Pferdebohne nicht wesentlich erhöht.

\section{Die Bestäubung der männlich-sterilen Pflanzen in verschiedenen Anbau-Anordnungen}

- Die Verbreiterung der Anbaustreifen von 2,5 $\mathrm{m}$ auf 7,5 $\mathrm{m}$ hat keinen Einfluss auf Bestäubung und Ertrag : der Fruchtansatz in den Mittelstreifen schwankt zwischen 5,2 und 15,1 Hülsen je Stengel $(\varnothing=7,5)$ in den breiten Streifen $(7,5 \mathrm{~m})$ und zwischen 5,3 und 11,1 $(\varnothing=7,5)$ in den schmalen Streifen $(2,5 \mathrm{~m})$. Die Übereinstimmung der Ergebnisse ist vielleicht auf die beträchtliche Wiederherstellung der männlichen Fruchtbarkeit innerhalb des weiblichen Stammes zurückzuführen (durchschnittlich $20 \%$ ).

\section{Schlussfolgerung}

Hortobombus, B. lapidarius und Eucera sind unter unseren Versuchsbedingungen in der Mehrzahl der Fälle für die Hybridsamen verantwortlich. Es erscheint nicht rentabel, Apis mellifica in diesem Anbau einzusetzen und zwar wegen ihres Verhaltens den männlich-sterilen Pflanzen gegenüber, bei den von Hummeln angestochenen Blüten und den mit Vicia faba konkurrierenden Pflanzen.

Man erwägt, Felder zur Produktion von Hybridsamen in solchen Gebieten anzulegen, die reich an Hummeln sind, ohne jedoch zu grosse Flächen anzubauen, um eine Verdünnung der wilden Fauna zu vermeiden. 


\section{RÉFÉRENCES BIBLIOGRAPHIQUES}

Berthelem P. Communications personnelles.

Bond D. A., Hawkins R. P., 1967. Behaviour of bees visiting male-sterile field beans (Vicia faba). J. Agric. Sci. Camb., 68, 243-247.

Brandenburg W., 1961. Broad beans : causes of poor yields sought. N. Z. J. Agric. 102, $277-280$.

Brian A. D., 1954. The foraging of bumblebees. Bee World, 35, 61-67; 81-91.

Brian A. D., 1957. Differences in the flowers visited by four species of bumblebees and their causes. J. Anim. Ecol., 26, 69-96.

Delaude A. Données non publiées.

Delaude A., Audy J. M., 1970. La production de graines de féverole. Bull. Inf. F.N.A.M.S. Oct. 1970, 1-24.

FreE J. B., 1962. The behaviour of honeybees visiting field beans (Vicia faba). J. Anim. Ecol., 31, 497-502.

FreE J. B., 1965. The effect on pollen collection of feeding honeybee colonies with sugar syrup. J. Agric. Sci., 64, 167-168.

Free J. B., 1970. Insect pollination of crops. London - New-York. Academic Press 555 p.

Maurizio A. et Louveaux J., 1965. Pollens de plantes mellifères d'Europe. U.G.A.P. 38 Bd., Sebastopol Paris.

Percival M. S., 1955. The presentation of pollen in certain angiosperms and its collection by Apis mellifica. New. Phytol., 54, 353-368.

Poulsen M. H., 1971. Mode de travaux et rôle de l'abeille (Apis L.) et des Bourdons (Bombus Latr.) dans la production de graines chez la féverole (Vicia faba L.). Thèse Copenhague (en Danois).

Poulsen M. H., 1973. The frequency and foraging behaviour of honeybees and bumblebees on field beans in Denmark. J. Apic. Res., 12, 75-80.

Pritsch G., 1971. Recherches sur le rôle que joue l'abeille dans la pollinisation de la fève (Vicia faba). Congr. Intern. Apic. 23. Moscou. C.R. p. 529.

Scriven W. A., Cooper B. A., Allen H., 1961. Pollination of field beans. Outl Agric., 3, 69-75.

Steucka Rdt R., 1965. Pollensammelnde Honigbienen (Apis mellifica) als wirksame Bestäuber bei der Züchtung und im Samenbau von Luzerne, Rotklee und Ackerbohnen. Der Züchter 35, 66-72.

Wafa A. K., Монамed M. I., 1970. Anthophorinae pollinating on broad bean flowers. Bull. Soc. Entomol., Egypte, 54, 212-220. 